\title{
La théorie de la polyphonie et des stéréotypes au service de l'analyse contrastive des opérateurs discursifs
}

\author{
Carlos MELÉNDEZ QUERO \\ Université de Lorraine \\ carlos.melendez@univ-lorraine.fr \\ http://orcid.org/0000-0003-0619-948X
}

\section{Resumen}

Este trabajo profundiza en las equivalencias de hélas en español y se inscribe en el marco de los enfoques comparativos recientes ligados a las teorías lingüísticas de la enunciación. El objetivo del artículo es abrir una vía para el análisis contrastivo de los operadores discursivos que combine las propiedades sintácticas con un enfoque semántico que permita mostrar las similitudes y diferencias entre las instrucciones ligadas a su significado. El estudio ofrecido, ilustrado con ejemplos del corpus Frantext, refuerza la hipótesis de que un marco metodológico inscrito en la Teoría de los estereotipos y en la Teoría de la polifonía puede tener aplicaciones muy interesantes en la búsqueda de relaciones entre operadores afines de una misma lengua y de lenguas diversas.

Palabras clave: enfoque comparativo, argumentación, enunciación, estrategia discursiva, puntos de vista.

\section{Résumé}

Ce travail approfondit les équivalences de hélas en espagnol et s'inscrit dans le cadre des approches comparatives récentes liées aux théories linguistiques de l'énonciation. L'objectif de l'article est d'ouvrir une voie à l'analyse contrastive des opérateurs discursifs qui combine les propriétés syntaxiques avec une approche sémantique permettant de montrer les similitudes et les différences entre les instructions attachées à leur signification. L'étude proposée, illustrée avec des exemples issus du corpus Frantext, renforce l'hypothèse qu'un cadre méthodologique inscrit dans la Théorie des stéréotypes et la Théorie de la polyphonie peut avoir des applications très intéressantes dans la recherche des relations entre opérateurs proches d'une même langue et de langues différentes.

Mots-clés : approche comparative, argumentation, énonciation, stratégie discursive, points de vue.

\footnotetext{
*Artículo recibido el 14/08/2020, aceptado el 12/02/2021.
} 


\begin{abstract}
This work delves into the equivalences of hélas in Spanish and is part of the recent comparative approaches linked to the linguistic theories of enunciation. The aim of the article is to open a way for the contrastive analysis of the discursive operators that combines the syntactic properties with a semantic approach that allows showing the similarities and differences between the instructions linked to their meaning. The study offered, illustrated with examples from the Frantext corpus, reinforces the hypothesis that a methodological framework inscribed in the Theory of Stereotypes and the Theory of Polyphony can have very interesting applications in the search for relationships between related operators of a same language and different languages.
\end{abstract}

Keywords: comparative approach, argumentation, enunciation, discursive strategy, points of view.

\title{
1. Introduction
}

Buridant (2003 : 183) clôturait son article sur l'interjection ${ }^{1}$ en français et notamment sur l'analyse de hélas, en expliquant que son étude « demanderait des affinements, des compléments et des prolongements vers d'autres langues romanes ». De même, Świątkowska (2006 : 55) considérait «hors de doute que le fonctionnement discursif " de ces unités « est toujours à décrire » et esquissait l'importance de sa comparaison dans des langues différentes.

Suivant cette piste, le travail de Meléndez Quero (2019) ouvre une première voie pour la comparaison de hélas vers d'autres langues, fondée sur des données tirées de corpus écrits et à partir de l'analyse des propriétés syntactico-distributionnelles, permettant de montrer ses ressemblances et divergences avec ses équivalents en espagnol. L'étude ici présentée ${ }^{2}$ donne suite à une des voies encore non exploitées pour l'analyse contrastive de hélas, qui est celle d'aborder ses choix de traduction en espagnol par l'étude des instructions discursives et des phrases stéréotypiques qui sont attachées à la signification de cet opérateur.

Dans cette optique, nous démarrerons cet article visant les avantages d'une approche contrastive renouvelée (section 2). Puis, après un rappel diachronique sur la formation de hélas (section 3) et son fonctionnement syntaxique en synchronie (section

\footnotetext{
${ }^{1}$ Sur les problèmes des critères définitoires de cette catégorie ou sa considération en tant que partie du discours, nous renvoyons à la monographie de Buridant (2001 : en ligne), ainsi qu'aux travaux de Rosier (1995: $110 ; 1997: 451$ et $2000: 19)$, Bertin (2002 : en ligne), Buridant (2006:3-9), Demonet (2006 : 59), Kleiber (2006: 10), Sierra Soriano (2006: 73), Świątkowska (2006: 47-49), Vincensini (2006: 101) ou Riegel, Pellat et Rioul (2009: 771-774), entre autres. Dans le cadre de l'espagnol, $c f$. Real Academia Espańola (2009 : 2480-2481).

${ }^{2}$ Cette étude s'inscrit dans le cadre des travaux de l'ATILF-CNRS et du projet P18-FR2619 Macrosintaxis del discurso persuasivo: construcciones y operadores, financé par la Junta de Andalucía et dont les chercheuses responsables sont Catalina Fuentes Rodríguez et Ester Brenes Peña.
} 
4), nous aborderons en détail l'étude de hélas en tant qu'opérateur discursif (section 5) dans une démarche comparative inscrite dans un cadre scientifique alliant les apports de la théorie de la polyphonie et des stéréotypes dans le but d'expliquer les stratégies discursives qui sont attachées à l'emploi des éléments décrits.

\section{Le renouveau de l'approche contrastive}

Comme l'indique Rodríguez Somolinos (2011 : 7), «le terme contrastif est à comprendre de façon plurielle». Ainsi une approche contrastive peut "porter sur des marqueurs apparemment voisins dans deux langues différentes, ou bien, elle peut opposer deux ou plusieurs marqueurs à l'intérieur d'une même langue. Elle peut comprendre également la mise en contraste des différentes étapes dans l'évolution d'un marqueur".

Même si l'étude ici proposée rappelle les traits principaux de la formation et évolution de hélas en diachronie, elle vise notamment l'analyse synchronique de l'opérateur hélas à partir d'une double approche contrastive permettant d'établir des liens en français avec malheureusement, ainsi qu'avec ses choix de traduction en espagnol ${ }^{3}$.

Nous soutenons dans cet article l'intérêt de la linguistique dite contrastive pour la description des phénomènes de nature monolingue. Cette approche va dans le sens de Donaire (2019 : 34) : "On soutient aujourd'hui que la perspective contrastive permet de découvrir des aspects particuliers caractérisant une langue qui n'auraient pas pu être découverts par une recherche exclusivement monolingue $»^{4}$.

Notre étude comparative peut s'inscrire dans le cadre du renouveau de l'approche contrastive esquissé par Donaire (2019 : 28-37), marqué par l'accès aux grands corpus électroniques afin d'avoir un échantillon représentatif de données, par l'intérêt croissant des travaux portant sur la perspective comparée des unités discursives et par l'essor des théories de la linguistique de l'énonciation.

L'étude proposée ici suit une approche contrastive s'inspirant de la Théorie de l'argumentation dans la langue (Anscombre et Ducrot, 1983), ainsi que des apports plus récents "mettant en œuvre la théorie des stéréotypes et la polyphonie », théories qui "adoptent une conception dynamique de la langue, et se donnent comme précepte

\footnotetext{
${ }^{3}$ Face à une approche monolingue, la conclusion de Delahaie (2011: 130) sur le couple heureusement/malheureusement reconnait l'intérêt de l'analyse contrastive, laquelle " offre bien de pistes de recherche " et pourrait permettre de voir comment certaines langues romanes comme l'espagnol ou l'italien peuvent traduire de façon différente ces deux adverbes et notamment la construction heureusement que du français ; cet aspect a été abordée récemment par Anscombre (2017), qui souligne l'existence d'une matrice lexicale ayant subsisté dans ces langues romanes.

${ }^{4}$ L'approche contrastive peut avoir des conséquences positives dans le cadre de l'enseignement des langues étrangères et de la traduction ( $c f$. Meléndez Quero, 2016). Elle a également une application directe dans le renouveau d'une lexicographie bilingue (Donaire, 2019: 32).
} 
d'étudier la langue en situation, c'est-à-dire, les énoncés dans la situation d'énonciation» (Donaire, $2019: 29$ ).

Sans vouloir nous attarder sur des questions terminologiques ${ }^{5}$ qui dépasseraient les objectifs de cette contribution, nous soulignons que l'ancrage de cet article dans ces théories énonciatives nous amène à choisir le terme opérateur discursif ${ }^{6}$ pour référer au fonctionnement de hélas et ses équivalents.

En effet, la notion d'opérateur peut s'appliquer à un ensemble d'éléments de nature catégoriale diverse (adverbes, locutions, interjections, etc.) et «fait référence à une fonction qui est commune à tous ces éléments : instruire des opérations sémanticopragmatiques qui ont pour but la construction du sens de l'énoncé » (Vázquez, 2019: 689).

Avant de nous pencher sur les opérations discursives instruites par la signification de hélas qui constitue l'objet central de ce travail, nous rappellerons dans les pages qui suivent son procès de formation et évolution diachronique (section 3$)^{7}$, ainsi que ses propriétés syntaxiques en français contemporain (section 4).

\footnotetext{
${ }^{5}$ Comme l'indique Rodríguez Somolinos (2011 : 3), les études consacrées aux unités discursives manquent « de cohérence en ce qui concerne la définition des notions et la terminologie ». Nous renvoyons aux travaux de Anscombre (2018 : 40-43), Donaire (2018 : 13) ou Vázquez (2019: 683-689) remettant en question quelques étiquettes employées dans la littérature scientifique (connecteurs, marqueurs de discours, particules).
}

Nous rappelons que le terme partículas a été très emprunté en espagnol (Martín Zorraquino, 1992, 1999 et 2010 ; González Ruiz et Llamas Sáiz, 2011) et a servi d'inspiration aux dictionnaires spécialisés (Santos Río, 2003 ; Briz, Pons et Portolés, 2008 : en ligne). Par ailleurs, l'étude de l'interjection « s'est retrouvée prise dans l'intérêt plus général pour [...] les particules discursives " (Rosier 2000 : 19) ou parmi les particules de modalité (Martín Zorraquino, 1999 : 52 ; Buridant, 2006 : 5). Dans le cadre des études sur l'espagnol, le fonctionnement discursif des interjections permet à Real Academia Española (2009: 2480) de les regrouper sous l'étiquette des marcadores discursivos. Quelle que soit la nomenclature choisie, ces termes ne font pas allusion à une catégorie morphologique ou grammaticale, mais à une fonction discursive ( $c f$. Martín Zorraquino, 2010 : 93).

Enfin, des auteurs comme Bertin (2002 : en ligne), Świątkowska (2006 : 47-49) ou Martín Zorraquino (2010 : 96-97 et 131) considèrent des interjections en tant que signes. $C f$., dans ce sens, Kleiber (2006 : 16), pour qui en prononçant Aïe! ou Hélas! «nous sommes déjà au niveau de langage ou, autrement dit, nous avons déjà affaire à un signe linguistique ".

${ }^{6}$ Il s'agit d'un terme courant dans les travaux fondés sur des cadres scientifiques en lien avec les théories des stéréotypes et de la polyphonie, comme le montrent les ouvrages récents de Anscombre, Donaire et Haillet (2013) ou Anscombre, Haillet et Donaire (2018). Notons également que dans le domaine de l'espagnol le terme operador est utilisé dans le dictionnaire de Fuentes Rodríguez (2018).

${ }^{7}$ Nous soutenons l'utilité de la diachronie en complément de l'analyse en synchronie pour l'étude des opérateurs discursifs, choix qui a déjà été souligné par Rodríguez Somolinos (2011: 8), Anscombre (2016 : 222) ou Donaire (2018: 17) ; nous remarquons, à ce titre, l'étude de Delahaie (2011: 114) visant l'analyse diachronique " pour éclairer les disparités sémantiques et syntaxiques en synchronie entre heureusement et malheureusement", adverbes pouvant partager un fonctionnement discursif avec hélas. Par ailleurs, Donaire (2019: 35) rappelle que le renouveau des travaux contrastifs s'intéresse à « jumeler 


\section{Parcours diachronique : la formation et l'évolution de hélas en tant qu'opérateur discursif}

L'étude diachronique de hélas s'avère intéressante car elle permet d'éclaircir quelques liens avec las, ainsi qu'avec malheureusement et heureusement, qui nous semblent importants dans le cadre d'une approche contrastive de cet opérateur vers l'espagnol.

Tout d'abord, nous rappelons que hélas est composée de hé et de l'adjectif las au sens ancien de "malheureux", comme le témoignent les entrées de hélas dans le Trésor de la Langue Française informatisée (désormais TLFi) ou la neuvième édition du Dictionnaire de l'Académie française (en ligne).

Pour expliquer sa naissance et formation, nous partirons de Anscombre (1979: 82-83 et 1985 : 176) et Buridant (2003 : 174-175), auteurs qui considèrent hélas un délocutif formulaire.

En effet, selon Buridant (2003: 174), las "a comme étymon le latin lassus 'épuisé, accablé de fatigue', donnant en ancien français l'adjectif las, au sens de 'fatigué, d'abord, puis aussi de 'malheureux, misérable' ». Puis, entrant dans " la sphère du malheur et de la douleur qu'on en ressent ", les signes ha et hé "s'associent à las pour exprimer la douleur avec retour sur le sujet». Enfin, par « un processus de délocutivité formulaire, le figement autour de la forme au masculin, dont témoignent les manuscrits, fait perdre à las au sens de 'malheureux' son statut d'adjectif et, à l'instar de hé ! Iha !, las et hélas deviennent des interjections" (Buridant, 2003 : 175).

Anscombre (1979: 83) analyse hélas comme délocutif formulaire (diachronique), à partir d'un processus comprenant plusieurs étapes : dans la première, on aurait " deux morphèmes : le premier, hé, sert à attirer l'attention; le second est l'adjectif las = 'malheureux'"; puis, il y aurait la formule "hé, las! servant à se plaindre. On attire l'attention du destinataire à l'aide de hé, puis on lui déclare qu'on est las (malheureux) "; dans un troisième temps, suivrait la création d'un morphème complexe (hé-las), qui aurait «la valeur sémantique $S^{*}=$ l'acte qu'on fait en disant (hé, las!)"; enfin, les emplois de la formule hé, las! seraient relus en donnant cette valeur sémantique. Ainsi, « en disant hélas! je dis ce que je fais à seule fin de le faire ».

Par ailleurs, Anscombre (1985: 176) mentionne l'existence en français d'un délocutif lexical synchronique sous la forme d'un substantif (un hélas), qui est cependant " peu courant de nos jours » et qui est même considéré comme " familier " par la huitième édition du Dictionnaire de l'Académie Française, comme le rappelle Buridant (2001:37).

l'analyse synchronique avec la recherche diachronique » dans une même étude. Enfin, nous citons l'étude de Anscombre (2017) sur le que médiatif, lequel confirme l'intérêt des études combinant une double perspective (diachronique et comparée). 
En ce qui concerne l'évolution diachronique, les travaux de Buridant (2003: 175) et Vincensini (2006: 109) permettent d'apprécier que hélas est entré dans le domaine de la douleur morale, de l'affliction et de la déploration face à un malheur, puis se fige progressivement "avec de moins en moins de possibilité de disjonction ", avec une soudure de hé et las ayant pu "déjà se produire en moyen français ». L'alternance entre las et hélas dans le domaine de l'imploration, la douleur et le regret se maintient encore au $\mathrm{XVI}^{\mathrm{e}}$ siècle, puis au XVII ${ }^{e}$ le vieillissement de las comme interjection s'accentue et hélas devient de plus en plus utilisé comme interjection plaintive. Selon le parcours tracé par Buridant (2003 : 176), cette évolution diachronique se serait consommée en arrivant à la distribution actuelle, où l'usage interjectif de las serait qualifiée de archaïque ou vieilli par les dictionnaires du français (Robert, 2010 : 1433 ; TLFi : en ligne), restant l'emploi adjectif de las, qui "se maintient dans la sphère de la fatigue, de la lassitude, de l'accablement"; hélas aurait concurrencé las "dans la sphère de la douleur " et l'aurait marginalisé " jusqu'à en faire un résidu archaïque »" si bien, "il serait réducteur de le limiter à la seule expression de la plainte ", aspect dont nous reviendrons plus en détail lors de la présentation des instructions discursives attachées à la signification de hélas.

Par ailleurs, l'étude de la formation de hélas permet d'établir quelques liens avec malheureusement, adverbe crée sur la base féminine de l'adjectif malheureux. Pour cela, nous partons de Anscombre (1980), qui analyse quelques similitudes et différences entre hélas, malheureusement et la formule c'est malheureux que. Selon cet auteur, " hélas marque un acte de se plaindre, dérivé d'un acte primitif du style de $p$ est malheureux ", à différence de malheureusement et de c'est malheureux que, qui " ne réalisent cet acte de plainte que de façon allusive " (Anscombre, 1980 : 114). Cet acte primitif « serait donc une espèce de trace laissée par la diachronie - au niveau de l'illocutoire, au moins - de la signification initiale de hélas, qui était quelque chose comme Dis donc, c'est malheureux» (Anscombre, $1980: 113$ ).

L'analyse de hélas comme marqueur de dérivation d'un acte de plainte et l'explication de cet acte primitif sous la forme $p$ est un fait malheureux expliquent la possibilité de hélas d'entrer dans le fonctionnement de malheureusement comme adverbe de phrase ou adverbe disjonctif d'attitude (attitudinal disjunct, selon Greenbaum, 1969), que nous développerons dans la section 4.

Cette similitude avec les adverbes de phrase justifie que hélas entre dans des tournures avec des ajouts du type pour \{moi, toi, lui, elle, etc. $\}$, lesquels «oblitèrent

\footnotetext{
${ }^{8}$ Si Grevisse et Goosse (2008 : 1415) affirment que l'emploi interjectif de las « a retrouvé de la vitalité " en ce début du XXI siècle, les données de Frantext consultés en date du 6 juillet 2020 ne laissent entrevoir que 6 cas de las comme interjection exclamative datés au XXI ${ }^{\text {e }}$ siècle (contrairement aux 748 registres de hélas pour cette période, dont 161 avec intonation exclamative).
} 
l'acte dérivé " et montrent que " tout aspect signifiant n'a pas disparu de Hélas " (Anscombre, 1985 : 114-116), facilitant ainsi des enchaînements avec heureusement ${ }^{9}$ :

[1] La gauche a gagné, hélas pour toi et heureusement pour moi, car j'ai gagné mon pari (Anscombre, 1980 : 113).

L'aperçu diachronique sur la formation de hélas que nous venons de présenter permet d'envisager un fonctionnement syntaxique particulier de cet opérateur discursif en français pouvant faciliter, dans le cadre d'une approche contrastive, des équivalences avec des interjections, ainsi qu'avec des adverbes et locutions adverbiales du type disjonctif de l'espagnol.

\section{Les propriétés syntaxiques de hélas en synchronie}

Avant de nous pencher sur les instructions sémantico-discursives attachées à hélas qui permettent d'éclaircir les similitudes et différences avec ces opérateurs de l'espagnol, il nous semble pertinent de rappeler d'abord ses principaux traits syntaxiques en synchronie facilitant la comparaison avec des opérateurs proches. En effet, dans cette recherche, nous soutenons, tout comme Rodríguez Somolinos (2011 : 8, note 4), l'idée que si une étude scientifique partant des travaux de l'Argumentation dans la langue « est essentiellement centrée sur les propriétés sémantiques, elle n'exclut pas l'examen des propriétés syntaxiques ».

Buridant (2003 : 176), Grevisse et Goosse (2008 : 468), ainsi que le dictionnaire TLFi (en ligne) accordent à hélas la possibilité d'entrer dans un fonctionnement disjonctif comme élément incident dans une distribution partagée avec les adverbes évaluatifs du type malheureusement ${ }^{10}$, comme nous illustrons avec l'exemple [2], tiré de la Base textuelle Frantext ${ }^{11}$ :

\footnotetext{
${ }^{9}$ Le recours à l'approche diachronique permet à Delahaie (2011: 128-129) de montrer que, contrairement à malheureusement - dont l'emploi comme adverbe de phrase est très ancien -, la formation de heureusement comme adverbe de phrase n'a pas suivi le même parcours et pourrait s'expliquer - tout comme dans le cas de hélas - par un processus de délocutivité formulaire, cette fois-ci à partir des marqueurs de dérivation du type il \{est arrivélarrive\} heureusement que $p$. Ainsi, selon cette auteure, dans ces constructions, heureusement servirait dans un premier temps «à réaliser de manière allusive un acte de contentement : "Il est arrivé de manière heureuse que p, et je m'en réjouis" ». Puis, le passage à cet acte de réjouissance se ferait par une loi discursive du type "qualifier un événement d'heureux revient à se réjouir de cet événement ".

${ }^{10}$ Bertin (2002 : en ligne) reconnaît que hélas « serait proche d'un adverbe de phrase » et rappelle que cette idée de rapprocher l'interjection des adverbes de phrase n'a rien de très original, si on se réfere "à la tradition grecque qui ne distinguait pas ces formes des autres adverbes ".

${ }^{11}$ Cette étude est fondée sur un corpus récupéré de la base textuelle Frantext, dans sa version intégrale 19.4 et sur un filtre d'exemples du XXI ${ }^{e}$ siècle. Dans cette recherche nous avons fait le choix d'un corpus de référence pour l'étude de la langue française comme celui de Frantext, car il constitue un outil de recherche lemmatisé et catégorisé très performant et qui intègre une importante production de textes modernes et contemporains, visant l'emploi du français écrit incluant des textes littéraires, mais également, dans une moindre proportion, des textes scientifiques. Rappelons que le recours à un grand corpus
} 
[2] Je sais, hélas, que cette guerre a fait des millions de victimes

(Frantext : Lydia Flem, Lettres d'amour en héritage, 2006).

Ce fonctionnement correspond à l'emploi habituel de malheureusement ou heureusement en français parmi les adverbes disjonctifs d'attitude (Mørdrup, 1976 ; Molinier et Levrier, 2000).

L'emploi disjonctif de hélas comble en partie le manque d'adverbes formés sur les bases des adjectifs évaluatifs d'orientation défavorable de la langue française (regrettable, disgracieux, triste, lamentable) ayant pu développer cette fonction ( $c f$. Meléndez Quero 2018 et 2019) et présente hélas comme alternative à malheureusement en tant qu'adverbe disjonctif d'évaluation émotive négative ${ }^{12}$.

Du point de vue des propriétés distributionnelles, dans ces emplois disjonctifs, hélas partage avec l'adverbe malheureusement ou la locution adverbiale par malheur - ainsi qu'avec les équivalents en espagnol, comme desgraciadamente, desafortunadamente, lamentablemente, tristemente, por desgracia, etc. - la liberté positionnelle, en tête, fin ou incise dans la proposition, comme l'indique le travail de Buridant (2003 : 176) ou la description de hélas en fonction de "commentaire affectif " du TLFi (en ligne).

Rappelons également que, preuve de son lien avec les adverbes disjonctifs, hélas peut accepter une complémentation avec pour, que nous avons illustré avec l'exemple [1]. D'un point de vue contrastif, ces distributions avec ajout de pour sont acceptées

électronique enrichi du français comme Frantext a été soutenu, entre autres, par Donaire (2019 : 29). Par ailleurs, nous avons décidé d'étudier le français écrit, en suivant les recommandations de Rosier (2000 : 45) ou Hummel (2012 : 325), qui confirment l'intérêt de l'emploi de corpus de préférence écrite pour l'analyse de l'interjection et des adverbes énonciatifs, lesquels gardent des affinités avec le fonctionnement de hélas en français. Enfin, d'un point de vue contrastif, l'utilisation de Frantext permet également une comparaison la plus homogène possible avec d'autres corpus électroniques de référence de préférence écrite de l'espagnol comme CREA et CORPES XXI ; ainsi, nous renvoyons aux données déjà fournies dans Meléndez Quero (2019 : 590-591) quant à la fréquence des opérateurs discursifs à évaluation émotive dans ces trois corpus - Frantext pour le français, CREA et CORPES XXI pour l'espagnol permettant de étudier les préférences des francophones et des hispanophones au XXIe siècle.

${ }^{12}$ À différence de malheureusement, hélas n'a pas développé la possibilité de fonctionner comme un adverbe de manière, adjoint du verbe (cf. Mørdrup, 1976 : 199 ; Schlyter, 1977 : 198-202 ; Molinier et Levrier, 2000 : 52-53). Ce type d'emploi - qui dépasse les objectifs de cette recherche -, est très rare de nos jours dans le cas de malheureusement, ce qui le distingue des adverbes comme lamentablement. Par ailleurs, l'étude de Delahaie (2011 : 108-109) fondée sur corpus confirme que, contrairement à heureusement, malheureusement est très peu utilisé comme adverbe de constituant et que l'antonyme de heureusement en tant qu'adverbe de constituant ne serait pas malheureusement mais l'adverbe mal; Delahaie (2011 : 118-128) montre également que, du point de vue diachronique, heureusement a prédominé en tant qu'adverbe constituant avant qu'une apparition progressive en tant qu'adverbe de phrase ait eu lieu au XVIII ${ }^{e}$ siècle, tandis que l'emploi de malheureusement comme adverbe de manière aurait été une formation résiduelle et moins habituelle que son usage en tant qu'adverbe de phrase, dont l'apparition est ancienne. 
par malheureusement et favorisent la traduction de hélas par des adverbes disjonctifs de l'espagnol suivis de para (desgraciadamente para él), mais ne semblent pas acceptables par des interjections du type ay ou vaya.

Or, à différence de malheureusement, hélas peut garder une intonation exclamative dans les emplois disjonctifs, y compris en " rupture d'un syntagme verbal " (Buridant, 2003 : 176), comme l'on peut voir dans [3] :

[3] Je partage le point de vue de mon amie philosophe Annie

Leclerc, qui fut ma camarade de classe et qui nous a hélas !quittés cette année (Frantext : Macha Méril, Un jour, je suis morte, 2008).

Toujours dans une optique contrastive vers l'espagnol, ces distributions exclamatives ne sont pas acceptées par les adverbes évaluatifs du type desgraciadamente et favorisent la traduction de hélas par des interjections telles que jay! ${ }^{13}$ ou jlástima!

En effet, comme indiqué par Meléndez Quero (2019 : 593-594), le lien de hélas avec ay permet de repérer une série de propriétés communes, qui ne sont pas partagées par les adverbes disjonctifs du français et de l'espagnol. Ainsi, rappelons que, outre la possibilité d'admettre un emploi substantivé - peu habituel en français contemporain ${ }^{14}$-, hélas se distingue de malheureusement par la possibilité d'être suivi par des expressions exclamatives, ainsi que d'être dupliqué ou, même, triplé. Toutes ces distributions empêchent la traduction de hélas par des adverbes ou de locutions du type desgraciadamente ou por desgracia et facilitent le rapprochement avec des interjections comme ay.

Enfin, la traduction par ay est également conseillée dans les distributions de hélas en exclamation suivie d'adresse à Dieu, qui rappelle les expressions $A y$, Dios ou Ay, Señor (cf. Fuentes Rodríguez et Alcaide Lara, 1996 : 292) :

[4] Hélas! qu'un tel exil, Seigneur, me serait cher! (Frantext : Jacques Roubaud, Nous, les Moins-que-Rien, Fils aînés de Personne: $12(+1)$ autobiographies, 2006).

\footnotetext{
${ }^{13}$ Real Academia Espańola (2009 : 2494-2497) admet l'apparition des interjections (ay, oh, hala, etc.) à l'intérieur de la phrase servant à exprimer des réactions émotives. Or, comme l'indique Martín Zorraquino (1999 : 52) ces dernières ont une plus grande autonomie, notamment du point de vue suprasegmental, avec des pauses plus marquées et une intonation généralement exclamative. Rappelons que l'exclamation marque "un relief singulier " (Buridant, $2003: 176)$ et est signe de monstration de l'attitude du locuteur (Anscombre, $2018: 29-30$ ).

${ }^{14}$ Face à hélas, Anscombre (1985: 177) reconnaît que aïe n’a pas donné lieu de nos jours à un dérivé substantival; d'un point de vue contrastif, la situation en français differe de l'espagnol, où on aurait une interjection exclamative pleine $(; A y !)$ avec une fonction signifiante au-delà de la seule expression de la douleur physique, ainsi que l'existence d'un délocutif lexical substantif (un ay), équivalent ainsi aux emplois rares de hélas comme substantif.
} 
Outre l'adresse religieuse, la distribution de hélas dans [4] permet de repérer un schéma prosodique qui caractérise le fonctionnement de heureusement face à malheureusement. En effet, Anscombre (2016 : 207) et Delahaie (2018:258) confirment que heureusement autorise un schéma du type adverbe, pause, que $p$, pouvant même admettre certaines répétitions associées à des marques d'amplification (Heureusement, mais heureusement que $p$ ).

Or, notre étude fondée sur corpus confirme que la distribution hélas, pause, que $p$ est certes très rare en français, avec seulement deux cas dans Frantext au XXI siècle. D'ailleurs, nous n'avons trouvé aucun exemple dans le corpus étudié qui permettrait d'illustrer l'entrée de hélas dans les distributions du type heureusement que $p$, lesquelles ne sont pas acceptées par malheureusement ${ }^{15}$.

Si hélas n'a pas développé cette structure avec que, cette unité peut être employée seule, tout comme heureusement. En effet, Buridant (2013 : 176) ou le dictionnaire TLFi (en ligne) reconnaissent que hélas peut avoir un emploi interjectif - comme "mot-phrase ", selon la terminologie de Grevisse et Goosse (2008 : 1413) -, que nous repérons dans [5] :

[5] - Si elle avait quitté le casino, sans un sou, elle était peut-être échaudée pour longtemps... Mais l'avez-vous bien observée quand son numéro est sorti ?

- Hélas!(Frantext : Marcel Aymé, Nouvelles complètes, 2002).

Si hélas partage avec heureusement la possibilité d'apparaître seul dans un dialogue comme réponse à une question ou à une assertion préalable ${ }^{16}$, nous soutenons

\footnotetext{
${ }^{15}$ Le couple adverbial heureusement/malheureusement a été traité dans de nombreux travaux (Mørdrup 1976 ; Schlyter, 1977 ; Guimier, 1998 ; Molinier et Levrier, 2000 ; Culioli, 2001 ; Furukawa, 2005 ; Delahaie, 2011 et 2014 ; Anscombre, 2016 et 2017; Meléndez Quero, 2009b et 2018, entre autres), visant à expliquer l'acceptabilité de heureusement que contrairement à *malheureusement que en français. Sans pouvoir nous attarder sur cette question, nous renvoyons à Anscombre (2016:213), qui souligne la présence d'un que d'antériorité énonciative et pour qui le trait de l'asymétrie de heureusement, face à la symétrie de malheureusement « est à l'origine de la possibilité de heureusement que $p$ face à l'impossibilité de malheureusement que $p$ ".

${ }^{16}$ Rappelons que, face à l'acceptabilité de heureusement employé seul, Delahaie (2011 : 116) émet des réserves quant à l'acceptabilité de malheureusement dans ces distributions, en expliquant que cet adverbe "fonctionne beaucoup mieux en association avec oui ".

Par ailleurs, face à malheureusement, hélas peut apparaître seul comme réaction à une assertion ( $c f$. Delahaie, 2011 : 129). Cette dernière distribution est acceptable en français pour heureusement, ce qui confirme que «contrairement aux apparences, heureusement n'est pas le pendant négatif de malheureusement " (Anscombre, $2017: 4$ ). Le fonctionnement de heureusement se rapproche de celui de hélas, aspect que confirme les affinités de heureusement avec les énoncés exclamatifs et les interjections ( $c f$. Delahaie, 2011 : 129). Enfin, d'un point de vue comparatif avec l'espagnol, ces emplois seuls avec intonation exclamative ne sont pas acceptés par des adverbes évaluatifs comme afortunadamente ou desgraciadamente et restent cependant possibles avec des interjections du type ay ou lástima, ainsi que dans des répliques avec la locution menos mal.
} 
que le fonctionnement " autonome " de hélas ne l'est, en réalité, qu'en apparence ${ }^{17}$. En effet, en tant qu'opérateur discursif, la signification de hélas doit être toujours comprise par rapport à d'autres formes de discours (explicites ou implicites). Ainsi, dans [5], hélas introduit une évaluation défavorable sur un rôle discursif ayant été considéré antérieurement, qui est facilement déductible par le contexte, comme réponse affirmative à la question précédente (Hélas, je l'ai bien observée... ${ }^{18}$.

En résumé, l'étude des propriétés syntaxiques de hélas confirme un fonctionnement particulier permettant d'établir des affinités et des différences avec des opérateurs proches du français et de l'espagnol.

D’une part, hélas partage avec malheureusement ou heureusement et les adverbes évaluatifs de l'espagnol (desgraciadamente, desafortunadamente, lamentablemente, tristemente, etc.) un emploi en disjonction comme commentaire affectif, avec liberté positionnelle et acceptabilité de la complémentation avec pour (para en espagnol), distribution également possible avec les locutions adverbiales du type par malheur ou por desgracia. Or, face à malheureusement et aux adverbes et locutions de l'espagnol comme desgraciadamente ou por desgracia, hélas admet également l'intonation exclamative dans les emplois disjonctifs, caractéristique partagée par ¡ay! ou jlástima!

D'autre part, tout comme malheureusement et les adverbes de l'espagnol, hélas ne semble pas avoir entré dans la construction avec que (*hélas que...), ayant été développé par heureusement ou dommage en français, ainsi que par lástima en espagnol. Cependant, hélas se rapproche de heureusement et s'éloigne de malheureusement et des adverbes de l'espagnol comme desgraciadamente dans un emploi seul comme réponse à une question ou commentaire à une énonciation antérieure, souvent associé à une intonation exclamative, ce qui permet de rapprocher son fonctionnement aux interjections ay, vaya ou lástima en espagnol.

Enfin, malheureusement et les adverbes évaluatifs de l'espagnol du type desgraciadamente n'ont pas développé les distributions de hélas suivi d'autres expressions exclamatives ou d'adresses à Dieu, ni les emplois en duplication ou triplement, distributions que, tout comme l'emploi substantivé, favorisent plutôt le rapprochement de hélas avec l'opérateur ay en espagnol.

\footnotetext{
${ }^{17}$ Rosier $(1995$ : 113) critique ce critère d'autonomie absolue : « Or cette croyance en l'autonomie syntaxique absolue de l'interjection apparât comme un leurre au vu de la distribution particulière du motphrase en tête ou queue de proposition et des possibilités d'incises ». Bertin (2002 : en ligne) se demande si « est-on sûr que dans la réalité du discours, il y ait tant d'interjections à l'état libre, qui ne se raccrochent pas à une suite ?", et rappelle que "les emplois autonomes de I sont rares et particuliers ». Par ailleurs, l'emploi interjectif de hélas, employé seul, " comme réaction à un élément de la situation ", est considéré vieux et littéraire par le dictionnaire TLFi (en ligne).

${ }^{18}$ Hélas peut également être combiné à non dans une réponse négative à une question préalable. D’un point de vue contrastif, cette distribution est possible par malheureusement, ainsi que par les adverbes disjonctifs équivalents de l'espagnol (desgraciadamente, desafortunadamente, etc.).
} 
Une fois rappelé le fonctionnement syntaxique de hélas permettant de le rapprocher des adverbes disjonctifs et des interjections, nous allons à présent expliquer comme hélas partage quelques instructions sémantico-discursives avec des opérateurs sémantiquement proches, aspect qui aidera à éclaircir davantage les multiples choix de traduction de hélas en espagnol.

\section{Instructions sémantico-discursives attachées à bélas}

En tant qu'opérateur discursif à valeur clairement énonciative, l'utilisation de hélas constitue un choix du locuteur ${ }^{19}$. Ainsi l'indique Rosier (2000:22) : "Dire hélas correspond à un recours à l'institué de la langue plutôt qu'à une improvisation expressive ". Afin d'illustrer cet aspect, nous voudrions nous servir de l'exemple [6], tiré également du corpus Frantext :

[6] La sonnerie stridente de mon réveil l'arrachait au petit matin de l'inconscience, qui semblait désormais son climat favori, pour enfiler des vêtements de pêche encore humides et se traîner jusqu'au bateau qui, hélas pour lui, se dandinait sagement sur son mouillage.

Je dis « hélas» car j'ai compris beaucoup plus tard que Ploc avait peut-être tenté, la dernière année, d'échapper à la corvée de pêche (Frantext : Benoîte Groult, Mon évasion, 2008).

Comme le montre la deuxième apparition de hélas - celle qui suit le verbe dire -, nous sommes face à un opérateur linguistique choisi par le locuteur au moment de l'énonciation. Afin de comprendre ce choix du locuteur, nous devons tout d'abord nous attarder sur la «structure sémantique de base» (Sierra Soriano, $2006: 86$ ) permettant d'expliquer la signification de hélas, "indépendamment du contexte dans lequel elle est employée " (Świątkowska, 2006 : 48). Pour décrire ce qui caractérise la signification de hélas au niveau de la langue, nous allons inscrire l'analyse de cet opérateur dans un cadre polyphonique, précisé par la suite.

\subsection{Approche polyphonique pour l'analyse de hélas}

Suivant Donaire (2018: 12), nous prenons comme cadre "celui qui nous semble s'adapter le mieux à notre objet d'étude, à savoir, une version récente de la

\footnotetext{
19 Sur les problèmes de caractérisation des rôles appliqués à la notion du locuteur nous renvoyons à Anscombre (2018: 39), auteur qui reconnaît que "la distinction entre ces différents rôles dans des énonces complexes est loin d'être évidente, et n'a donné lieu, à notre connaissance, à aucune étude spécifique permettant d'établir des critères opératoires d'identification du locuteur et de ses différentes manifestations ». Dans cette contribution, nous partirons d'une approche du locuteur en tant qu'entité discursive que l'énoncé présente ou désigne comme son auteur.
} 
théorie de l'argumentation dans la langue ${ }^{20}$ qui incorpore, notamment la notion de stratégie discursive, la théorie des stéréotypes et la polyphonie".

Dans le but d'expliquer la signification de hélas, nous partons tout d'abord d'une approche polyphonique rejetant l'unicité du sujet parlant et facilitant la mise en scène de plusieurs voix au niveau discursif. Plus particulièrement, notre analyse se rapproche d'une version radicale de la polyphonie (Anscombre, 1990 ; Donaire, 2006), qui considère que "tout énoncé est polyphonique dès le niveau profond " et que "les entités 'objectales', du moins en apparence, ne réfèrent à rien d'autre qu'à un faisceau de discours $»^{21}$ (Anscombre, $2018: 22$ ).

D'après cette optique, nous faisons l'hypothèse que l'information sémantique inhérente à hélas ne peut s'expliquer que par une polyphonie ou dialogue entre plusieurs points de vue ${ }^{22}$ au niveau de la langue elle-même ${ }^{23}$.

Cette approche polyphonique est compatible avec la notion opérateur discursif choisie pour nous référer à hélas dans cette contribution. En effet, Vázquez (2019: 689) précise que l'étiquette "opérateur vise le rôle dynamique de ces unités et le caractère essentiellement actif des opérations réalisées. Ces opérations, qui sont générées dans les niveaux les plus profonds de la langue, contribuent à la constitution de stratégies discursives diverses ${ }^{24}$. Rappelons, à cet égard, qu'on peut parler de stratégie discursive " là où le discours met en relation deux (ou plusieurs) points de vue, l'objectif étant invariablement de déterminer quel procédé a pour résultat une telle mise en relation, et quels sont les points de vue mis en rapport» (Haillet, $2013: 34$ ).

\footnotetext{
${ }^{20}$ Les travaux d'Anscombre et Ducrot (1983) sur l'argumentation linguistique se situent dans une « sémantique discursive ascriptiviste, non référentialiste et de type instructionnel » (Rodríguez Somolinos, 2011 : 3). Dans cette recherche, nous partons de l'hypothèse que ce cadre scientifique est compatible avec une approche contrastive permettant de comparer les instructions attachées à des opérateurs proches de différentes langues, aspect également remarqué par Donaire (2019:29) : "Quant aux principes de Ducrot et Anscombre, même si la théorie n'exige pas l'étude simultanée de plusieurs langues, ils sont parfaitement transposables d'une langue à d'autres" ".

${ }^{21}$ Le point de départ de cette polyphonie radicale considère que " tout énoncé consiste en l'attribution de rôles discursifs aux personnages du discours - ce sont les fameuses voix -, et en une certaine organisation de ces rôles discursifs, le tout se situant au niveau de la structure de surface ", et que ces rôles et leur organisation « sont le résultat de l'interprétation d'une structure abstraite affectée à l'énoncé considéré » (Anscombre, $2018: 25)$.

${ }^{22}$ Nous suivons Delahaie (2014: 227, note 7), qui affirme " qu'un point de vue correspond à un rôle discursif et qu'il se décompose sous la forme d'un contenu linguistique associé à une source ».

${ }^{23}$ Donaire (2006 : 67-68) fait « référence à des dynamiques polyphoniques qui constituent la signification des mots de la langue, auxquelles ils ne peuvent pas échapper lors de leur énonciation ».

${ }^{24}$ Comme l'indique Donaire (2018: 13), les «stratégies discursives mettent en jeu des ressources linguistiques dont notamment des structures argumentatives et des structures polyphoniques reliant des points de vue ».
} 
Ce cadre scientifique posé, nous allons déterminer ce qui constitue la signification de hélas d'un point de vue polyphonique.

Pour cela, nous partirons de la description de Buridant (2003 : 177) à propos de la modalité exprimée par hélas, qui « est fondamentalement déroute, déroute par rapport à un attendu ».

Cette caractérisation permet de relier la signification de hélas à celle de malheureusement, qui « est fondamentalement le signe d'une absence de congruence entre une valeur, envisagée ou souhaitée, et une autre valeur, celle qui se trouve vérifiée par les faits " (Guimier, 1998 : 174). En effet, hélas et malheureusement partagent la possibilité d'introduire une évaluation défavorable par rapport à un rôle discursif que nous appellerons point de vue (pdv), généralement explicite, qui est regretté et qui est contraire à un autre point de vue (explicite ou implicite) qui est souhaité. Afin d'illustrer cet aspect, nous partirons des exemples qui suivent :

[7] Hélas, Jean rate son bac.

[8] Malheureusement, Jean rate son bac.

D’après une optique polyphonique visant la structure sémantique profonde, la signification de base de l'opérateur discursif hélas est constituée - tout comme celle de malheureusement - de l'interaction entre deux points de vue d'orientation contraire, que nous appellerons $p$ et $p$, montrant le premier $\{p\}$ ce qui est regretté ou lamenté \{Jean-rater-son-bac\} car en contradiction à ce qui est souhaité, et $p$ ' le point de vue opposé, c'est-à-dire, celui qui est souhaité ou attendu, mais n'ayant pas lieu \{Jean-avoirson-bac\} ${ }^{25}$.

En même temps, le choix de hélas - comme celui de malheureusement- implique un positionnement du locuteur face à ces deux points de vue opposés : une orientation défavorable par rapport à $p$ et une orientation favorable du pdv contraire envisagé $\{p\}$, auquel il s'oppose. Ainsi, la représentation de cette structure serait la suivante :

[Hélas/Malheureusement], $p$ (pdv défavorable) \{Jean-rater-sonbac\}, $p$ ' (pdv favorable, opposé à $p$ ) \{Jean-avoir-son-bac\}.

La signification polyphonique de base de hélas et de malheureusement requiert une dynamique dialogique excluant le point de vue favorable $p^{\prime}$ - qui reste généralement implicite - et sélectionnant le point de vue défavorable $p$, celui qui apparaît

${ }^{25}$ S'agissant de représenter une structure profonde, nous suivons le modèle de Anscombre (2018: 26) qui formule les $p d v$ sous la forme $\{\mathrm{O}\}$, où $\mathrm{O}$ est le contenu ou objet-construit, et fait le choix d'une formulation avec le verbe à l'infinitif "n'ayant d'autre but que d'éviter la confusion entre langue et métalangue ». Rappelons que, dans le cadre choisi, ces contenus ou objets construits «seraient des "phrases" de la métalangue, qui lors de l'interprétation et de l'instanciation des rôles discursifs, deviendraient des énoncés réels ou virtuels " (Anscombre, 2018 : 26, note 12). Ainsi, ces phrases de la métalangue en structure profonde seraient parfaitement récupérables en structure de surface par des énoncés du type Jean rate son bac. 
normalement au niveau de l'énoncé et qui fait allusion à ce qui est contraire à ce qui est souhaitée ${ }^{26}$.

Notons que ce schéma polyphonique distingue hélas ou malheureusement de heureusement, dans un contexte identique :

[9] Heureusement, Jean a son bac.

Si la signification de heureusement ne peut s'expliquer que par l'interaction entre deux points de vue opposés, la stratégie discursive impliquée par le choix de cet adverbe implique la sélection $\mathrm{du}$ pdv d'orientation favorable souhaité et qui a lieu ${ }^{27}$ \{Jean-avoir-son-bac\} et, en même temps, l'exclusion d'un autre pdv (envisagé) contraire aux attentes du locuteur ${ }^{28}$ \{Jean-rater-son-bac $\}$. Ainsi, la représentation de la structure de base à partir de [9] serait la suivante :

Heureusement, $p$ (pdv favorable) \{Jean-avoir-son-bac\}, $p^{\prime}(\mathrm{pdv}$ défavorable, opposé à $p$ ) \{Jean-rater-son-bac\}.

En reprenant le cas de hélas et suivant le modèle de Delahaie (2018), nous pouvons préciser que, si la structure polyphonique de cet opérateur présente $p$ comme argumentativement opposé à $p$ ', cela implique nécessairement que les conclusions ou conséquences que l'on peut tirer de $p$ et de $p$ ' sont également opposées, ce qui ferait intervenir l'interaction de deux autres points de vue. Ainsi, dans un exemple comme [7], hélas présente une orientation défavorable de $p$ qui aboutirait à une conclusion $r$ \{Jean-ne-pas-accéder-à-l'université\}; cette conclusion serait contraire à celle que l'on

\footnotetext{
${ }^{26}$ L'analyse polyphonique présentée ici est compatible avec les explications de Anscombre (1980:111), qui décrit les liens entre hélas et malheureusement en affirmant que tous les deux ne commentent pas directement un énoncé, mais le présentent comme contraire à un autre (explicité ou non) qui pourrait s'envisager. Or, cet auteur souligne une différence importante entre ces deux opérateurs, car malheureusement implique un changement entre deux orientations argumentatives opposées, qui n'est toujours pas nécessaire dans le cas de hélas. En effet, à différence de malheureusement, la présence de hélas ne requiert pas que l'énoncé antérieur ait obligatoirement une orientation argumentative contraire à celle introduite par $p$.

${ }^{27}$ Heureusement se distingue de malheureusement par la congruence entre ce qui est souhaité et la réalité. Cf. Guimier (1998), Culioli (2001), Furukawa (2005) ou Delahaie (2011 et 2014).

${ }^{28}$ La configuration polyphonique de base de heureusement est partagée par des locutions du type par chance ou par bonheur, qui sélectionnent l'orientation discursive à sens favorable (celle représentant le point de vue souhaité), contrairement à dommage et aux locutions évaluatives d'orientation défavorable comme par malchance ou par malheur, caractérisées - tout comme hélas ou malheureusement - par le trait d'absence de congruence entre valeur envisagée et réalité. D'un point de vue contrastif, le schéma polyphonique sélectionnant le point de vue souhaité permet d'associer heureusement aux adverbes du type afortunadamente ou des locutions comme por suerte ou por fortuna, tandis qu'une structure polyphonique excluant le point de vue attendu ou souhaitée et sélectionnant le point de vue contraire aux attentes du locuteur - comme celle de hélas et malheureusement - est partagée par les adverbes desgraciadamente, desafortunadamente, lamentablemente, etc., des locutions comme por desgracia, ainsi que par des interjections comme lástima, ay ou vaya. Pour une analyse détaillée des dynamiques polyphoniques inhérentes aux adverbes d'évaluation émotive de l'espagnol, $c$. Meléndez Quero (2009a).
} 
pourrait tirer de $p^{\prime}$, conclusion non-r dont le contenu ou objet construit qu'on peut représenter en structure profonde aurait la forme \{Jean-accéder-à-l'université\}. Ainsi, le locuteur qui introduit hélas évalue défavorablement $p$ en fonction de la conclusion $r$ que l'on peut tirer de $p$.

Afin de clore cette analyse polyphonique de hélas, il faut préciser que hélas introduit cette évaluation défavorable en vertu d'un principe général (ou norme) selon lequel « on doit avoir le bac pour accéder à l'université », principe qui, dans un contexte par défaut, est censé être reconnu et accepté par la communauté linguistique à laquelle le locuteur appartient. Cette norme, qui renvoie à la notion de phrase stéréotypique dont nous reviendrons dans la sous-section qui suit, introduit ainsi un nouveau point de vue mis en jeu, dont la source est un ON-locuteur (Anscombre, 2005), communauté dont le locuteur fait partie, et l'objet construit est \{on-devoir-avoir-le-bac-pour-accéder-àl'université\}.

En somme, suivant le modèle de Delahaie (2018), on pourrait gloser hélas de la manière suivante: "Quand on énonce hélas, $(p)^{29}$, le locuteur qualifie de défavorables les conclusions $r$ que l'on pourrait tirer de $p$ au nom d'un principe général, et il met à distance les conclusions non-r que l'on pourrait tirer de $p^{\prime} »^{30}$. Cette analyse confirme notre hypothèse selon laquelle la signification de hélas s'explique par une polyphonie ou interaction entre plusieurs points de vue.

\subsection{Approche stéréotypique pour l'analyse contrastive de hélas}

L'analyse polyphonique de hélas a montré que l'évaluation défavorable de $p$ instruite par cet opérateur marque en même temps la non-conformité à une norme sémantique ou principe général (" on doit avoir le bac pour accéder à l'université ", dans l'exemple antérieur), qui fait intervenir la notion de stéréotype et « qui a bien sûr

${ }^{29}$ Cette glose nous semble également valable dans les cas où hélas est employé seul dans des répliques ; bien entendu, dans ce type de cas $p$ reste implicite, mais est facilement récupérable à partir d'une énonciation antérieure. Rappelons, à cet égard, notre hypothèse selon laquelle le fonctionnement autonome de hélas ne l'est qu'en apparence, car la signification de cet opérateur discursif implique nécessairement l'interaction avec d'autres rôles discursifs, souvent implicites.

${ }^{30} \mathrm{Du}$ point de vue de la modélisation polyphonique, dans [7], hélas met en jeu six points de vue :

$$
\begin{aligned}
& \operatorname{Pdv}_{1}: p\{\text { Jean-rater-son-bac }\} \\
& \mathrm{Pdv}_{2} \text { : conclusion } r \text { de } \mathrm{pdv}_{1} \text { \{Jean-ne-pas-accéder-à-l'université\} } \\
& \mathrm{Pdv}_{3}: p^{\prime}\{\text { Jean-avoir-son-bac }\}
\end{aligned}
$$

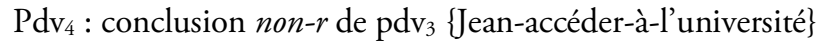

$$
\begin{aligned}
& \mathrm{Pdv}_{5} \text { : norme (stéréotype) à partir de laquelle le locuteur évalue comme défa- } \\
& \text { vorable } \mathrm{pdv}_{1} \text { et } \mathrm{pdv}_{2} \text { \{on-devoir-avoir-le-bac-pour-accéder-à-l'université\} } \\
& \mathrm{Pdv}_{6} \text { : évaluation défavorable }\left\{\text { hélas\} de } \operatorname{pdv}_{1} \text { et } \operatorname{pdv}_{2} \text { par rapport à } \mathrm{pdv} \mathrm{v}_{5}\right.
\end{aligned}
$$

En ce qui concerne le positionnement du locuteur sur ces pdv, on pourrait dire qu'il s'identifie et prend

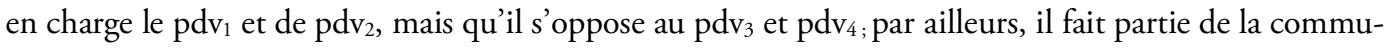
nauté linguistique (ON-locuteur) qui est la source $\mathrm{du}_{\mathrm{pdv}}$; enfin, il est la source du pdv6 qui constitue l'évaluation défavorable $\mathrm{de} \mathrm{pdv}_{1}$ et $\mathrm{pdv}_{2}$ en vertu $\mathrm{de} \mathrm{pdv}_{5}$. 
des liens très forts avec la représentation du monde développée par une communauté linguistique donnée" (Delahaie, $2011: 115)$.

Cette notion renvoie à la théorie des stéréotypes ( $c f$. Anscombre, 1995 et 2001 ; Donaire, 2006), selon laquelle le stéréotype associé à un terme est constitué d'une liste ouverte des phrases attachées à chaque mot, lesquelles sont censées être acceptées par une communauté linguistique ${ }^{31}$.

Suivant Donaire (2006 : 69), nous soulignons l'intérêt de cette approche qui vise à compléter l'analyse polyphonique " avec une autre dynamique qui instruit le dialogue-mot phrases stéréotypiques, qui précise l'orientation défavorable et les discours possibles ».

Nous pencher sur les phrases stéréotypiques inhérentes à la signification de hélas nous permettra ainsi de préciser son lien avec d'autres unités du lexique et les formes de discours auxquelles l'opérateur fait allusion. Notre hypothèse est que l'analyse des phrases stéréotypiques attachées à hélas permet, d'un point de vue contrastif, d'affiner ses ressemblances et différences par rapport à d'autres opérateurs de l'espagnol avec lesquels il partage des instructions discursives ${ }^{32}$.

Afin d'ouvrir une nouvelle voie pour l'analyse des phrases stéréotypiques attachées à hélas et s'agissant d'un opérateur à valeur clairement énonciatif, nous nous servons d'un métalangage pour expliquer le mécanisme discursif activé par le choix de hélas, que nous représentons sous la forme suivante:

"Dire hélas, (p) c'est dire p... ».

Dans cette représentation, nous utilisons le verbe être afin de présenter une équivalence entre deux propositions introduites par un verbe de langue (dire) : la première inclut hélas et le point de vue $p$, généralement explicite ${ }^{33}$ et qui est orienté défavorablement ; la deuxième aide à expliciter comment la signification de hélas permet d'interpréter cette évaluation défavorable de $p$.

\footnotetext{
${ }^{31}$ Selon Anscombre (2001 et 2005), ces phrases stéréotypiques attachées en langue à chaque mot renvoient à une sorte de savoir partagé et connu à l'intérieur d'une même communauté linguistique (ONlocuteur). Cf., également, Świątkowska (2006 : 55), qui évoque l'idée du savoir partagé et d'une relation stéréotypique « déclenchée dans la mémoire du locuteur par un mécanisme mettant en marche le préconstruit » liée à la signification de l'interjection, composée elle-même d'une série d'instructions. Cela explique bien les associations habituelles à certaines émotions dans le cadre de l'utilisation et compréhension des interjections, comme rappelle Kleiber (2006: 17) à propos des signes Aïe! et Hélas!

${ }^{32}$ Si l'analyse polyphonique antérieure de hélas qualifiant de défavorables les conclusions $r$ que l'on pourrait tirer de $p$ et excluant les conclusions non-r que l'on pourrait tirer de $p$ ' (point de vue souhaité) permet de l'associer à des opérateurs évaluatifs négatifs de différent statut catégoriel (desgraciadamente, por desgracia, ay, vaya, etc.), l'analyse du stéréotype attaché à la signification qui constitue chaque unité linguistique permet de préciser les liens entre ces opérateurs proches.

${ }^{33}$ Ce modèle de représentation discursive ne différencie pas les distributions (en réplique, antéposé, insérée, postposée, etc.) de hélas par rapport à $p$, point de vue pouvant également être implicite.
} 
Une fois posé ce cadre méthodologique, nous décrivons par la suite les phrases stéréotypiques constitutives de la signification de hélas favorisant ses liens avec certains opérateurs évaluatifs en espagnol, aspect pour lequel nous prenons en compte également les descriptions de hélas dans les dictionnaires et les travaux de spécialité.

5.2.1. Dans un premier temps, hélas est rattaché au champ lexical « de la douleur» (cf. Buridant, 2003 : $177^{34}$; Littré : en ligne) et très souvent associé à l'affliction (cf. Académie française : en ligne), la peine (Ducrot, 1972), le chagrin (Bertin, 2002 : en ligne) ou la tristesse ( $c f$. Vanderveken, 1992: 23 ; Buridant, $2001: 14$; Demonet, 2006 : 65 ; Świątkowska, 2006 : 52-53 ; Fraisse et Paroubek, 2015 : en ligne). Cette valeur, partagée par malheureusement, ainsi que par les adverbes desgraciadamente, lamentablemente ou tristemente, la locution por desgracia ou les interjections ay et lástima de l'espagnol, peut être actualisée par des phrases stéréotypiques du type :

Dire hélas, ( $p$ ) c'est dire $p$ \{entraînelcauselimpliquelengendreldonne lieu à\} \{l'affliction, la peine, la tristesse, la douleur, la souffrance, le chagrin, etc. ${ }^{35}$.

L'exemple [10] illustre cette valeur où hélas introduit $p$ présentant un contenu non souhaité (la tumeur est bien cancéreuse) ${ }^{36}$, justifiant la lamentation :

[10] Hélas, la tumeur est bien cancéreuse et il va falloir m’opérer sans délai, cette tumeur ayant grossi de 4 millimètres en un mois (Frantext : Serge Doubrovsky, Un homme de passage, 2011).

Si bien ce texte montre le lien entre hélas et malheureusement, hélas peut ajouter une nuance expressive plus marquée par rapport à l'adverbe, évidente dans les exemples avec intonation exclamative, comme [11] :

\footnotetext{
${ }^{34}$ Buridant (2003 : 177) précise que « c'est une douleur morale et non physique, au regard de la douleur exprimée par aïe " ou d'autres signes du français comme ouille. Du point de vue contrastif, la forme ay - tout comme huy - peut exprimer en espagnol contemporain, autant la douleur physique (équivalente à iqué dolor.) que la douleur morale (équivalente à qqué pena !) (Porroche Ballesteros, 2009 : 193-200) ; cette double valeur explique que ay puisse être employé comme traduction de hélas et de aïe, interjection cette dernière n'ayant pas conservé dans son statut moderne la valeur de tristesse ou lamentation propre à hélas (cf. Rosier, 1997 : 458).

${ }^{35}$ Dans le cas des opérateurs de l'espagnol, on pourrait représenter ces phrases par des formules rédigées dans cette langue et introduites par le verbe decir: "Decir desgraciadamente, $(p)$ es decir $p$ \{conllevalcausalimplicalda motivo para\} \{la aflicción, la pena, la tristeza, el dolor, el sufrimiento, etc.\}. Cf. Meléndez Quero (2009a et 2015).

${ }^{36}$ Nous écrivons ici l'énoncé réel en structure de surface, à partir des phrases de la métalangue en structure profonde illustrant le point de vue avec l'objet construit \{la-tumeur-être-bien-cancéreuse\}. Par ailleurs, rappelons que ce point de vue s'oppose à p' souhaité \{la-tumeur-ne-pas-être-bien-cancéreuse\}, et présente $p$ comme défavorable en fonction d'un principe général ou d'une norme acceptée par la communauté linguistique qui est associé au stéréotype du mot tumeur, stéréotype selon lequel « une tumeur cancéreuse peut être mortelle ».
} 
[11] Hélas! Tout est perdu et je n'ai rien à espérer (Frantext : Marcel Aymé, Nouvelles complètes, 2002).

Pour Świątkowska (2006 : 52-53), «le sentiment est présenté non seulement au moyen de, mais aussi à travers l'énonciation dont il est l'origine prétendue ». D'après cette optique, l'énonciation de hélas semblerait étant provoquée, arrachée ou imposée par la propre émotion ${ }^{37}$ et cette énonciation se présenterait comme une prise de conscience, un effet ou une conséquence directe de la douleur, tristesse ou affliction, censées être éprouvées au moment de l'énonciation ${ }^{38}$.

L'intonation exclamative pour marquer la souffrance ou la tristesse favorise la traduction de hélas dans [11] par jay! Elle est également très présente dans les textes littéraires caractérisés par un ton de lamentation qui empreint l'énonciation, comme l'illustre l'exemple [12] :

[12] Hélas! Il est dur d'être seule et si sage à vingt ans, et de ne pas pouvoir nommer un seul ami parmi tant des visages ${ }^{39}$.

(Frantext : Mireille Havet, Journal 1918-1919, 2003).

Citons également le texte suivant, où la coloration de la voix caractéristique de hélas est décrite entre parenthèses :

[13] J'ai une triste nouvelle à vous annoncer, un grand malheur, hélas (avec un ton de voix sourd et cassé qui transmettait la vérité avant que les mots eux-mêmes ne disent l'irréparable), elle était

${ }^{37}$ Cette idée d'imposition, qui apparaît déjà dans les définitions de hélas dans TLFi (en ligne), pourrait préciser la formulation des phrases stéréotypiques précédentes comme suit :

Dire hélas, $(p)$ c'est dire $p$ \{entraînelcauselimpliquelengendreldonne lieu à\} \{l'af-

fliction, la peine, la tristesse, la douleur, la souffrance, le chagrin, etc.\}, émotion qui \{arrache/impose/est à l'origine de\} l'énonciation de hélas.

Cf. également Bertin (2002 : en ligne) à propos de la manière dont hélas « apporte un commentaire sur l'énoncé dans sa validité ou les conditions de son énonciation ", ainsi que de son approche de l'interjection qui « est un peu un adverbial mais c'est aussi un déictique qui inscrit la force vive de l'énonciation ». ${ }^{38}$ L'étude de Kleiber (2006 : 18) sur les interjections illustre ce point : « Elles sont des indices ou index en ce qu'au moment de leur émission, elles sont reliées de manière indexicale ou causale à l'émotion éprouvée par le sujet qui les prononce ». Fraisse et Paroubek (2015 : en ligne) présentent aussi les interjections comme «marqueurs de subjectivité et plus particulièrement d'émotion » et rappellent que les interjections «sont nécessairement les indices linguistiques d'une émotion ».

${ }^{39}$ Dans ce type d'emplois, nous pouvons trouver un lien sémantique entre hélas et las, adjectif appliqué à celui qui « éprouve une fatigue mentale, morale, affective ou psychique et en vient à ne plus supporter ou à supporter difficilement quelque chose ou quelqu'un» (TFLi : en ligne). Cet aspect confirme que malgré sa valeur discursive hélas n'est pas désémantisé, car maintient un lien avec las. Sur cet aspect, nous rejoignons Bertin (2002: en ligne) pour qui dans les interjections secondaires (dont l'ancienne las.) « la parenté perceptible avec un signe lexical d'une autre catégorie morphologique garantit un contenu propositionnel ». 
très malade depuis plusieurs jours (Frantext : Lydia Flem, Comment j'ai vidé la maison de mes parents, 2004).

Dans l'exemple antérieur, le lien avec l'affliction de hélas favorise son choix pour transmettre de l'empathie ou de la commisération à l'interlocuteur au moment de lui transmettre une mauvaise nouvelle ${ }^{40}$. Cet emploi est partagé malheureusement, qui nous semble commutable avec hélas dans [14] :

[14] Je m'inquiète de mes collègues haïtiens.

- Des nouvelles de l'université ?

- Oui, des bonnes et des mauvaises, hélas. À l'ENS, toute

l'équipe administrative se trouvait au troisième étage pour une réunion. À la deuxième secousse, le bâtiment s'est effondré (Frantext : Lionel-Édouard Martin, Le Tremblement : Haïti, 12 janvier 2010, 2010).

Tout comme malheureusement, le choix de hélas sert à exprimer de l'affliction ou de la pitié au moment d'annoncer une information contraire aux souhaits de l'interlocuteur ; cette fonction interactive empathique est également partagée par desgraciadamente, lamentablemente, tristemente, por desgracia, ay et lástima en espagnol, mais ne semble pas favorisée par la signification d'un adverbe comme desafortunadamente, qui évoque une plus grande indifférence ou insensibilité (au lieu d'une pitié ou compassion) par rapport à la souffrance de l'interlocuteur ( $c f$. Meléndez Quero, 2015 : 154155).

Or, si bien l'association de hélas à des contextes tragiques présente généralement son énonciation " comme provoquée par quelque chose comme de la tristesse " qui est supposée, pour cette raison, être éprouvée ou ressentie "au moment même où on l'énonce ", De Cornulier (2001 : 111) indique que " on peut l'employer froidement, l'écrire même » dans certains contextes non exclamatifs. Afin d'illustrer cet aspect, nous nous servons de l'exemple qui suit:

[15] Hélas, la belle-mère du maçon est morte la veille, on l'enterre le lendemain, en sorte qu'il manquera sa propre consécration (Frantext : Emmanuel Carrère, Un roman russe, 2007).

Hélas partage avec malheureusement la possibilité d'être employé par le locuteur comme commentaire négatif portant sur un point de vue $p$ introduisant un contenu faisant allusion à la mort d'un être humain (la belle-mère du maçon est morte la veille), décès qui ne semble pas se présenter comme une source d'affliction ou une tragédie en soi, mais simplement comme n'étant pas opportun, en tant que provoquant une conclusion négative explicitée dans une suite discursive (il manquera sa propre

\footnotetext{
${ }^{40}$ L'introduction de hélas n'a pas ici la valeur de je me plains, mais vise à plaindre le caractère malheureux de $p$ en marquant la compassion envers l'interlocuteur, avec un sens de j'en suis désolé pour vous. Cf. Anscombre (1980: 114).
} 
consécration $)^{41}$. Du point de vue de la comparaison avec l'espagnol, le distancement de la valeur expressive de tristesse, peine ou affliction rapprocherait ces emplois de hélas du choix des opérateurs discursifs desafortunadamente et vaya ${ }^{42}$.

5.2.2. Voyons d'autres emplois de hélas montrant que le rattachement au seul champ lexical de la douleur " ne suffit évidemment pas à le définir ", étant donné que " hélas est loin de se réduire à prendre la relève de aïe » et présente « un très large spectre [...] sous la modalité émotionnelle " (Buridant, 2003: 177 et 180).

Comme déjà précisé, la signification de hélas offre une évaluation défavorable par rapport à une forme discursive ou point de vue $p$, qui est contraire au point de vue $p$ ' attendu par le locuteur, lequel est normalement implicite, mais peut parfois être explicité dans une énonciation préalable ${ }^{43}$, comme dans l'exemple [16], où nous voyons un changement d'orientation argumentative, redoublé par la présence de mais, entre $p$ ' souhaité (je comptais rembourser avec les indemnités que devait me verser la société d'assurance) et $p$ non souhaité (ces indemnités n'arrivaient jamais) ${ }^{44}$ :

[16] [...] je comptais rembourser avec les indemnités que devait me verser la société d'assurance.

Mais, hélas! ces indemnités n'arrivaient jamais et j'ai dû faire plusieurs emprunts successifs (Frantext: Pauline Picquet, Sans illustration, 2013).

Si nous nous plaçons dans une perspective temporelle pour expliquer cet écart par rapport à ce qui est souhaité $\{p\}$, nous pouvons distinguer un écart par rapport à " ce qui était attendu dans le passé - c'est le regret - ou était attendu dans l'avenir - l'appréhension" (Buridant, 2003 : 177-178). Dans ce dernier cas, nous avons une déploration prospective, que nous illustrons ci-dessous :

[17] Hélas, j’ai devant moi mon plus dur chemin! Hélas, je commence ma course la plus solitaire! (Frantext : Colette Fellous, Avenue de France, 2001).

Afin de représenter cette valeur d'appréhension ou de crainte, reconnue dans les travaux de l'espagnol pour ay - expression de «temor ", selon le dictionnaire Lema

\footnotetext{
${ }^{41}$ Rappelons que, d'après l'approche polyphonique, hélas évalue défavorablement $p$ en vertu d'une conclusion $r$ (explicite ou implicite) que l'on peut tirer de $p$.

${ }^{42}$ Contrairement à ay, qui est spécialisé dans l'expression de la douleur et l'affliction, le choix de vaya implique une plus grande insensibilité (cf. Porroche Ballesteros, 2017: 188) et marque davantage de froideur par rapport aux énoncés relatifs au décès d'une personne.

${ }^{43} \mathrm{TLFi}$ (en ligne) reconnaît cet emploi pour hélas : « Introduit une séquence rapportant un point de vue nouveau (celui d'un personnage et/ou de l'auteur...), un fait en contradiction avec ce qu'attend le personnage dans la séquence précédente ".

${ }^{44} \mathrm{Si} \mathrm{l'opposition} \mathrm{entre} \mathrm{un} \mathrm{point} \mathrm{de} \mathrm{vue} \mathrm{souhaité} \mathrm{antérieur} \mathrm{et} \mathrm{le} \mathrm{point} \mathrm{de} \mathrm{vue} p$ évalué défavorablement par hélas explique la fréquence d'emploi de hélas précédé par mais (cf. Buridant, $2003: 176)$, il ne s'agit pas d'un type d'enchaînement nécessaire pour hélas, comme l'indique Anscombre (1980 : 111-122).
} 
de Battaner Arias (2001), ainsi que dans les études de Fuentes Rodríguez et Alcaide Lara (1996 : 283-284) et Edeso Natalías (2010 : 242) -, nous nous servons des phrases stéréotypiques du type :

Dire hélas, ( $p$ ) c'est dire $p$ \{entraînelcauselimpliquelengendreldonne lieu à\} \{la crainte, l'appréhension, etc. ${ }^{45}$.

Beaucoup plus fréquente que cette valeur prospective de crainte est le choix de hélas dans une perspective rétrospective pour marquer le regret ${ }^{46}$ quant à l'écart entre ce qui était souhaité dans le passé et ce qui a eu lieu au moment de l'énonciation de hélas, comme dans l'exemple [16] cité ou dans [18] :

[18] Mon cœur battait très vite; j'attendais un triomphe. Hélas ! je n'ai entendu que des hurlements ${ }^{47}$ (Frantext : Alice Prin, Souvenirs retrouvés (de Kiki de Montparnasse), 2005).

Ces emplois de hélas marquant le regret par rapport à une attente du passé permettent d'actualiser des phrases stéréotypiques du type :

Dire hélas, $p$ c'est dire (p) \{entraîne/causelimpliquelengendreldonne lieu à\}, \{la déception ${ }^{48}$, la frustration, la résignation, le regret, etc.\}.

Le choix de hélas pour orienter défavorablement $p$ montrant un résultat décevant, comme celui de [21], le rapproche des adverbes lamentablemente et tristemente de l'espagnol :

[19] Nous avons visité tous les établissements scolaires du quartier, hélas sans aucun résultat (Frantext: Marc Levy, L'étrange voyage de Monsieur Daldry, 2011).

La commutation par malheureusement étant possible dans ce type de contextes, il convient de rappeler que, contrairement à hélas, la signification de cet adverbe peut

\footnotetext{
${ }^{45}$ Bien entendu, l'évaluation défavorable de $p$ se fait par rapport à une série de conséquences négatives envisageables pouvant entrainer un malheur à l'avenir, conclusion $r$ défavorable pouvant être implicite et à laquelle le locuteur «s'attend, par prédiction " (Buridant, 2003 : 178).

${ }^{46}$ L'allusion au regret apparaît dans les entrées lexicographiques de hélas dans les dictionnaires Robert (2010 : 1223), Académie française, TLFi ou Littré (en ligne). Elle est aussi reconnue par Bertin (2002 : en ligne) - « interjection privilégiée [...] des regrets qu'inspirent les mauvaises actions passées »-, Buridant (2003 : 171) - «modalisation affective exprimant le regret » - ou Kleiber (2006: 20) - emploi provoqué par une situation « de regret».

${ }^{47}$ Cet exemple montre bien que, d'une perspective temporelle, le point de vue souhaité ou attendu $p$ ' (j'attendais un triomphe) est antérieur chronologiquement au point de vue $p$ regretté (je n'ai entendu que des hurlements).

${ }^{48}$ L'utilisation de hélas pour marquer la déception est reconnue dans le dictionnaire de l'Académie française (en ligne), ainsi que par Buridant (2003 : 178), auteur qui cite aussi la possibilité de marquer la frustration. Par ailleurs, les phrases stéréotypiques antérieures pourraient être représentées également sous la forme «Dire hélas, $(p)$ c'est dire p est \{décevant, frustrant, etc.\}».
} 
renvoyer à la malchance ou l'infortune, ce qui facilite un emploi visant la sauvegarde de l'image publique face à l'interlocuteur ; en effet, tout comme desgraciadamente ou por desgracia en espagnol, le lien de malheureusement avec la malchance d'éléments fortuits peut être exploité pour réduire la responsabilité personnelle face à un résultat décevant ( $c f$. Meléndez Quero, 2015: 155). Le lien de hélas avec la base adjective las semble renvoyer à une intention différente montrant la fatigue et la lassitude et surtout un sentiment de regret et frustration. Ainsi, le choix de hélas semble une alternative pouvant combler en français la faible utilisation des adverbes de phrase évaluatifs sur la base des adjectifs regrettable ou triste, case cependant bien remplie en espagnol par les adverbes lamentablemente et tristemente.

Les emplois de hélas montrant une valeur de déroute, regret et de lassitude face à des résultats décevants sont très fréquents en français :

[20] Hélas, ma paresse et mon tempérament velléitaire m'ont empêchée de transformer ce rêve en projet (Frantext: Pauline Picquet, Sans illustration, 2013).

Parfois, comme le montre ce dernier exemple, ces cas sont accompagnés d'une sorte d'autocritique quant à certains défauts ou limitations qui sont reconnus, avec un ton de désillusion et résignation ${ }^{49}$ :

[21] J'arrive difficilement à concevoir des histoires. Je n'ai pas l'imagination de Jules Verne ou d'Alexandre Dumas -hélas! (Frantext: Georges Perec, Entretiens et conférences I [19651978], 2003).

Ces intentions sont également présentes dans des interlocutions visant l'interlocuteur. Regardons, à ce titre, la réponse de [22] qui est contraire aux attentes de l'interlocuteur et en même temps source de frustration, ce qui facilite l'équivalence de hélas avec lamentablemente et tristemente:

[22] - «Vous êtes pianiste, je crois ?»

- "Hélas, madame, non", dus-je répondre à regret (Frantext :

Jonathan Littell, Les Bienveillantes, 2006).

Dans ces cas où l'information introduite par hélas est susceptible de décevoir l'interlocuteur, le choix de hélas peut être compris comme une formule lexicalisée

\footnotetext{
${ }^{49} \mathrm{Si}$ bien la valeur de désillusion, déception ou frustration n'est pas reconnue pour ay dans les dictionnaires monolingues de l'espagnol ni par Edeso Natalías (2010), il nous semble que dans un exemple comme celui-ci on pourrait aussi envisager un sens d'impuissance de hélas du type "pauvre de moi » (cf. Vincensini, 2006 : 110), proche de la formule ay (pobre) de mí en espagnol. Par ailleurs, quant à vaya, nous rappelons que la valeur de déception est citée dans le dictionnaire de la Real Academia Española (en ligne), celle de désillusion dans le dictionnaire de Moliner (1966) et celle de résignation dans Santos Río (2003 : 639) ; ces valeurs expressives sont parfois mélangées avec l'idée d'agacement, très caractéristique de vaya. Enfin, lástima peut également entraîner une valeur de regret et déception, que l'on retrouve dans la quatrième acception de dommage du dictionnaire de l'Académie française.
} 
d'excuse, comme le montre la précision se désola-t-elle du texte [23], favorisant également la traduction par lamentablemente :

[23] Hélas, je ne monte pas par-là, se désola-t-elle en lui indiquant le fond de la cour (Frantext: Anna Gavalda, Ensemble, c'est tout, 2004).

Lié à cet emploi nous retrouvons le recours à hélas pour annoncer une mauvaise nouvelle, contraire aux attentes de l'interlocuteur, comme celle de [24] :

[24] L'établissement de l'horaire auquel j'ai travaillé ces jours montre nettement, hélas, que le personnel est plus que suffisant pour l'année scolaire 1965-1966. Il est impossible de te donner un travail, même pour un demi-horaire (Frantext : Patrick Modiano, Un pedigree, 2005).

Cet exemple correspond à l'utilisation de hélas pour indiquer que « le locuteur n'énonce une phrase rapportant un fait que parce que ce fait s'impose à lui, et ceci bien qu'il regrette ou se plaigne (qu'il puisse regretter ou se plaindre) des conséquences possibles [...] pour quelqu'un d'autre " (TLFi : en ligne). Ainsi, le choix de hélas, qui peut être traduite par lamentablemente, constitue une formule lexicalisée semblable aux expressions je regrette ou j'ai le regret et permet au locuteur de jouer un rôle le montrant comme étant obligé et dans l'impossibilité d'annoncer une autre nouvelle à son interlocuteur que celle qui est censée le décevoir. Notons à nouveau que la commutation possible dans ces contextes par malheureusement pourrait ajouter une allusion à la malchance minimisant ainsi la responsabilité personnelle dans la décision prise, emploi partagé par desgraciadamente et por desgracia en espagnol, grâce aux liens avec la mala suerte.

5.2.3. Enfin hélas est souvent décrit dans les travaux de spécialité (Anscombre, 1985 : 176 ; Vanderveken, 1992 : 23 ; Buridant, 2003 : 176) ainsi que dans les dictionnaires de l'Académie française, TLFi (en ligne) ou Robert (2010 : 1223) avec une valeur de plainte.

Cette valeur est illustrée dans [25], où hélas est employé dans le but de se plaindre de ce qui est énoncé dans une suite discursive (ils ne représentent que de belles promesses) contredisant les attentes préalables:

[25] Eh oui ! nous les avons ces précieux tickets, bien enfermés dans des enveloppes de mica, mais hélas! Bien souvent, ils ne représentent que de belles promesses illusoires, des sortes de chèques sans provisions (Frantext: Berthe Auroy, Jours de guerre: Ma vie sous l'Occupation, 2008).

Le choix de hélas offre une orientation défavorable par rapport à $p$, présenté comme "déplaisant » ou "fâcheux ", et par conséquent, source possible de plainte et 
d'insatisfaction, contrariété ou mécontentement, comme nous illustrons par les phrases stéréotypiques suivantes, attachées à la signification de hélas :

Dire hélas, ( $p$ ) c'est dire $p$ est \{déplaisant, fâcheux, ennuyeux, gềnant, etc.\}.

Dire hélas, (p) c'est dire p \{entraîne, implique, engendre, suscite, donne lieu à\} \{la plainte, l'insatisfaction, le déplaisir, le mécontentement, l'ennui, la gêne, la contrariété, la colère, etc.\}.

$\mathrm{Du}$ point de vue contrastif, la contrariété qui arrache l'énonciation de hélas ${ }^{50}$ semble avoir une équivalence avec l'emploi de ay avec valeur de plainte ou protestation reconnue par Fuentes Rodríguez et Alcaide Lara (1996: 285), Porroche Ballesteros (2009 : 193) ou Edeso Natalías (2010 : 241 et 244). Par ailleurs, la Real Academia Española (2009 : 2483 et 2511) reconnaît également la valeur de contrariété à lástima. Enfin, cet emploi de hélas est partagé par vaya, dont la valeur de plainte et contrariété, accompagné parfois d'un ton d'irritation, semble incontournable, comme l'indiquent Fuentes Rodríguez et Alcaide Lara (1996 : 304-305), Santos Río (2003 : 639) ou Tangue $(2015: 130)^{51}$.

Partageant avec malheureusement l'expression de l'insatisfaction ( $c f$. TLFi : en ligne), hélas se retrouve souvent dans le corpus étudié sans exclamation dans un emploi servant à évaluer $p$ comme "fâcheux " ou "déplaisant " :

[26] La mort d'une librairie, c'était une catastrophe minuscule et, hélas, banale (Frantext : François Maspero, Les abeilles et la guêpe, 2002).

[27] Cette époque, hélas, où la collectivité primait, disparaît, peu à peu (Frantext : Hélène Castel, Retour d'exil d'une fermme recherchée, 2009) $)^{52}$.

Cette valeur critique l'associe aux adverbes lamentablemente et tristemente de l'espagnol, lesquels ont assuré - à différence des adverbes dérivés des bases lamentable

\footnotetext{
${ }^{50}$ La valeur d'ennui et contrariété (souvent soudaine) a été développée en français par aïe comme réaction à des surprises désagréables ( $c f$. Robert, 2010 : 54, ainsi que les dictionnaires de l'Académie et TLFi : en ligne). De même, TLFi (en ligne) reconnaît l'acception " chose fâcheuse » dans les emplois figés de dommage, les valeurs de contrariété, déception et colère pour mince! et également le sens de colère et irritation de $z u t$ !

${ }^{51}$ Cf. également les entrées de vaya dans les dictionnaires de Maldonado (1996) et Real Academia Española (en ligne), ou les entrées du verbe ir dans les dictionnaires de Moliner (1966) et de Seco, Andrés et Ramos (1999). Enfin, notons que vamos peut être également employée comme exclamation de plainte et mécontentement (face à anda et venga exprimant le refus), tout comme hala et d'autres expressions de la langue familière (cáspita, caramba, caray, carajo, joder, etc.), dont l'étude dépasse les objectifs de cette recherche.

52 Cet exemple montre que la plainte peut être associée à la nostalgie, valeur que Porroche Ballesteros (2009 : 195) concède à $a y:$ " expresa nostalgia, que es la queja por el tiempo que se ha ido ».
} 
et triste du français - un usage répandu (surtout le premier) en tant qu'adverbes disjonctifs. Ainsi, l'analyse contrastive confirme, une fois de plus, que hélas rempli ainsi un rôle pas assumé par les adverbes regrettablement, fâcheusement, tristement ou lamentablement, qui n'ont pas développé l'emploi disjonctif en français.

L'étude des exemples du corpus Frantext montre enfin que hélas peut parfois introduire quelques rôles discursifs de critique plus sévère, accompagnées d'une valeur dépréciative ou péjorative, comme illustrent les textes [28] et [29] :

[28] L'enseignement de la philosophie que j'étais censé y recevoir s'avéra, hélas, affligeant, jusqu'à l'absurde (Frantext : Didier Eribon, Retour à Reims, 2009).

[29] Hélas, elle n'a qu'une âme de grue, n'aime que l'argent, les robes, et le luxe qu'il procure (Frantext : Mireille Havet, Journal 1918-1919, 2003).

Ces exemples favorisent le rapprochement avec certains emplois péjoratifs de lamentablemente et lástima en espagnol et permettent d'actualiser des phrases stéréotypiques attachées à hélas du type :

Dire hélas, $(p)$ c'est dire $p$ est \{regrettable, déplorable, etc.\}.

Dire hélas, $(p)$ c'est dire $p$ \{suscitelinspirelmérite\} \{la réprobation,

la pitié, le mépris, etc.\}.

\section{Conclusion}

Cette contribution a illustré le renouveau de l'approche contrastive pour l'étude des opérateurs discursifs se donnant pour cadre scientifique la linguistique de l'énonciation et bénéficiant notamment des apports récents de la théorie de la polyphonie ${ }^{53}$ et de la théorie des stéréotypes.

S’inspirant de ce cadre théorique, cette étude, portant sur l'opérateur hélas et fondée sur un corpus d'exemples tirés de la base textuelle Frantext, a confirmé l'hypothèse de l'intérêt d'une approche méthodologique multiple pouvant combiner l'analyse des propriétés syntactico-distributionnelles et des opérations sémantico-discursives attachées à la signification, lesquelles " configurent des agencements particuliers définissant chaque unité et permettant d'établir des similitudes et des différences " (Donaire, 2018 : 13). L'analyse contrastive ici esquissée a ainsi démontré l'utilité d'ajouter à l'étude du fonctionnement syntaxique des opérateurs décrits l'analyse des instructions discursives, dans une approche qui bénéficie des apports de la théorie de la polyphonie et de la théorie des stéréotypes.

Suivant les approches contrastives qui essaient de jumeler les études synchroniques avec les données de la diachronie, ce travail a prouvé également les apports d'un

\footnotetext{
${ }^{53}$ Si Anscombre (2018 : 46) reconnaît que la version récente de la polyphonie « est certes très imparfaite ", il est important de remarquer, comme le souligne cet auteur, qu'en tant que théorie " elle remplit son rôle qui est de faire découvrir de nouveaux phénomènes et de nouvelles problématiques ».
} 
aperçu diachronique ayant servi à montrer les liens de hélas avec malheureusement et ayant permis d'envisager un comportement syntaxique facilitant des rapprochements avec les interjections et les adverbes disjonctifs, ce qui explique son ample choix de traductions vers l'espagnol.

Par ailleurs, l'analyse polyphonique de hélas a permis de vérifier l'hypothèse selon laquelle sa signification en langue requiert toujours une interaction entre différents points de vue, pouvant être implicites ou explicites au niveau de la structure de surface. Si le cadre de la polyphonie explique comment le choix de cet opérateur exige une dynamique discursive et un positionnement du locuteur par rapport à deux points de vue $(p$ et $p$ ) qui sont arguments de deux conclusions d'orientation contraire ( $r$ et non-r) en vertu d'une norme ou principe général, ce travail a confirmé l'intérêt de se pencher également sur les phrases stéréotypiques qui constituent l'information sémantique attachée à la signification de hélas, lesquelles nous semblent offrir une nouvelle perspective pour l'analyse contrastive de hélas permettant d'éclaircir ses affinités avec des entités lexicales de différent statut catégoriel (adverbes, locutions adverbiales et interjections) avec lesquelles il partage des instructions discursives.

Ainsi, cette recherche a confirmé l'hypothèse qu'un cadre scientifique fondé sur les apports de la théorie des stéréotypes est compatible avec une approche comparative. À partir d'une formulation des phrases stéréotypiques de hélas servant à illustrer le mécanisme énonciatif activé par cet opérateur, notre contribution a essayé d'ouvrir une voie encore peu exploitée mais qui s'avère très utile dans le cadre d'une démarche comparative, car elle aide à préciser ses équivalences avec quelques opérateurs discursifs proches de l'espagnol, tels que ay, vaya, lástima, por desgracia, desgraciadamente, lamentablemente, tristemente ou desafortunadamente.

Notre étude a confirmé que les phrases stéréotypiques présentant $p$ comme donnant lieu à l'affliction ou la tristesse permettent de rapprocher la signification de hélas avec celle de malheureusement, ainsi qu'avec celle des interjections ay ou lástima, de la locution por desgracia et des adverbes desgraciadamente, lamentablemente ou tristemente, opérateurs qui partagent des emplois dont la signification est exploitée dans une fonction interactive empathique de compassion avec la douleur de l'interlocuteur. Face à la douleur physique présentée par ä̈e, la signification de hélas renvoie à une douleur plutôt morale et, contrairement à malheureusement, elle peut être accompagnée d'une intonation exclamative, ce qui favorise son lien avec ;ay! en espagnol.

Le rapprochement de hélas avec desafortunadamente ou vaya en espagnol a lieu dans des contextes également acceptés par malheureusement, où ces opérateurs ne servent pas à présenter $p$ comme entraînant l'affliction, mais comme n'étant pas opportun car donnant lieu à une suite discursive défavorable.

Le stéréotype de hélas inclut, tout comme celui de ay, des phrases permettant de présenter $p$ comme impliquant la crainte ou l'appréhension envers ce qui est attendu à l'avenir. Et surtout, avec une perspective rétrospective, la signification de hélas 
actualise des phrases stéréotypiques qui présentent $p$ comme entraînant un regret par rapport à ce qui était attendu dans le passé ; si la commutation par malheureusement est possible dans ce type d'emplois, la signification de hélas ne comporte pas l'allusion à la malchance ou l'infortune inhérents à malheureusement et partagés en espagnol par desgraciadamente ou por desgracia, ni ne renvoie à l'absence de fortune propre à desafortunadamente. Face à ces derniers opérateurs, le lien sémantique avec la base adjective las explique l'emploi de hélas avec une valeur de déception ou frustration, partagée en espagnol par les adverbes lamentablemente et tristemente, ainsi que par lástima et vaya.

La valeur de plainte caractéristique de hélas, illustrée par des phrases stéréotypiques servant à présenter $p$ comme fâcheux ou déplaisant et donnant lieu à la contrariété ou mécontentement, est partagée par malheureusement et aïe (ainsi que par d'autres opérateurs proches comme dommage, mince ou zut) et permet d'établir des affinités avec ay, vaya ou lástima, ainsi qu'avec les adverbes tristemente et lamentablemente. Enfin, lamentablemente et lástima partagent avec hélas des emplois plus péjoratifs présentant $p$ comme regrettable.

Pour clore cet article, nous voulons souligner que ce regard contrastif avec l'espagnol contribue à faire apparaître la particularité de la langue française, dont la fréquence d'emploi de hélas comme opérateur servant à offrir une évaluation défavorable de $p$ a pu compenser l'absence d'adverbes à orientation négative qui auraient pu se spécialiser, comme malheureusement, dans cette fonction disjonctive, aspect dont la classe réduite du français diffère de l'éventail de choix d'adverbes disjonctifs de l'espagnol (desgraciadamente, desafortunadamente, lamentablemente, tristemente). Cette comparaison avec l'espagnol valide l'hypothèse de l'utilité de la perspective contrastive dans le but d'éclairer des aspects de nature monolingue, telle que l'utilisation fréquente en français de hélas ayant assumé un emploi disjonctif pas assuré par les adverbes regrettablement, fâcheusement, tristement, lamentablement et disgracieusement.

\section{RÉFÉRENCES BIBLIOGRAPHIQUES}

ACADÉMIE FRANÇAISE (en ligne) : Dictionnaire de l'Académie française. $9^{e}$ édition. URL: https://academie.atilf.fr/9.

ANSCOMBRE, Jean-Claude (1979) : «Délocutivité benvenistienne, délocutivité généralisée et performativité ». Langue française, 42, 69-84.

AnsCOMBRE, Jean-Claude (1980) : «Voulez-vous dériver avec moi ?». Communications, 32, 61-124.

ANSCOMBRE, Jean-Claude (1985) : «Onomatopées, délocutivité et autres blabas ». Revue Romane, $20: 2,169-206$. 
ANSCOMBRE, Jean-Claude (1990) : "Thème, espace discursif et représentation événementielle ", in Jean-Claude Anscombre et Gino Zaccaria (éds), Fonctionnalisme et pragmatique. A propos de la notion de thème. Milan, Unicopli, 43-150.

ANSCOMBRE, Jean-Claude (1995) : "Semántica y léxico: topoi, estereotipos y frases genéricas ». Revista española de lingüistica, 25 : 2, 297-310.

ANSCOMBRE, Jean-Claude (2001) : "Le rôle du lexique dans la théorie des stéréotypes ». Langages, 142, 57-76.

ANSCOMBRE, Jean-Claude (2005) : «ON-locuteur : une entité à multiples visages », in Jacques Bres, Pierre Patrick Haillet, Sylvie Mellet, Henning Nølke et Laurence Rosier (éds), Dialogisme, polyphonie : approches linguistiques. Bruxelles, De Boeck-Duculot, 75-94.

ANSCOMBRE, Jean-Claude (2016) : «Les constructions en adverbe que $p$ en français : essai de caractérisation sémantique d'une matrice lexicale ». Cahiers de lexicologie, $108: 1$, 199223.

ANSCOMBRE, Jean-Claude (2017) : "Le que médiatif du français contemporain. Perspective diachronique et comparée ». Revue romane, $53: 2,181-216$. DOI : https://doi.org/10.1075/rro.16022.ans.

ANSCOMBRE, Jean-Claude (2018) : «Représentation sémantique des opérateurs discursifs : polyphonie, médiativité et autres », in Jean-Claude Anscombre, Pierre Patrick Haillet et María Luisa Donaire (éds), Opérateurs discursifs du français, 2. Eléments de description sémantique et pragmatique. Berne, Peter Lang, 21-46.

ANSCOMBRE, Jean-Claude \& Oswald DUCROT (1983) : L'argumentation dans la langue. LiègeParis-Bruxelles, Mardaga.

Anscombre, Jean-Claude, María Luisa DONAIRE \& Pierre Patrick Haillet [éds] (2013) : Opérateurs discursifs du français. Eléments de description sémantique et pragmatique. Berne, Peter Lang.

Anscombre, Jean-Claude, Pierre Patrick Haillet \& María Luisa Donaire [éds] (2018) : Opérateurs discursifs du français, 2. Eléments de description sémantique et pragmatique. Berne, Peter Lang.

ATILF-CNRS \& UNIVERSITÉ DE LORRAINE (sd): Base textuelle Frantext. URL: https://www.frantext.fr.

ATILF-CNRS \& UNIVERSITÉ DE LORRAINE (sd) : Trésor de la Langue Française informatisé. URL : http://www.atilf.fr/tlfi.

BATTANER ARIAS, Paz (2001) : Lema: diccionario de la lengua española. Barcelone, Spes.

BERTIN, Annie (2002) : «Définir une interjection : la lexicographie au défi de l'énonciation ». Linx, 12. URL : http://journals.openedition.org/linx/1277.

BRIZ, Antonio, Salvador PONS \& José PORTOLÉS [coords.] (2008) : Diccionario de partículas discursivas del español. URL : http://www.dpde.es.

BURIDANT, Claude (2001 : en ligne) : L'interjection en français : esquisse d'une étude diachronique. Strasbourg, Université March Bloch. URL: http://buridantesque.fr/wp-content/uploads/interjection.pdf. 
BURIDANT, Claude (2003) : "L'interjection en français : esquisse d'une étude diachronique. Le cas de hélas ", in Fernando Sánchez Miret (coord), Actas del XXIII Congreso Internacional de Lingüistica y Filología Románica, vol. 2/1. Tubingue, Max Niemeyer Verlag, 169-184.

BURIDANT, Claude (2006) : «L'interjection : jeux et enjeux ». Langages, 161, 3-9.

Culioli, Antoine (2001) : «Heureusement!», in Maria Helena Mateus et Clara Nunes Correia (éds), Saberes no tempo - Homenagem a Maria Henriquez Costa Campos. Lisbonne, Colibri, 279-284.

De CORnUlier, Benoît (2001) : Tigres et autres problèmes de sémantique. Nantes, Centre d'analyse linguistique du discours, Université de Nantes, MSH Ange.

DELAHAIE, Juliette (2011) : « L'illusion synchronique et des leçons de l'histoire. Interprétation sémantique d'un curieux couple faussement symétrique, malheureusement/heureusement (que)". Revue de sémantique et de pragmatique, 29-30, 107-133.

DELAHAIE, Juliette (2014) : «Les constructions en $a d v$. que $p$ : étude diachronique d'une tournure particulière à partir du cas de heureusement que p ", in Jean-Claude Anscombre, Eveline Oppermann-Marsaux et Amalia Rodríguez Somolinos (éds), Médiativité, polyphonie et modalité en français. Paris, Presse de la Sorbonne Nouvelle, 223-241.

DELAHAIE, Juliette (2018) : "Entité lexicale : heureusement", in Jean-Claude Anscombre, Pierre Patrick Haillet et María Luisa Donaire (éds), Opérateurs discursifs du français, 2. Eléments de description sémantique et pragmatique. Berne, Peter Lang, 249-261.

DEMONET, Marie-Lucie (2006) : «Eh/hé : l'oralité simulée à la renaissance ». Langages, 161, 57-72.

DONAIRE, María Luisa (2006) : "Les dialogues intérieurs à la langue ». Le Français Moderne, $74: 1,61-73$.

DONAIRE, María Luisa (2018) : "Introduction ", in Jean-Claude Anscombre, Pierre Patrick Haillet et María Luisa Donaire (éds), Opérateurs discursifs du français, 2. Eléments de description sémantique et pragmatique. Berne, Peter Lang, 11-18.

DONAIRE, María Luisa (2019) : «L'approche contrastive aujourd'hui en linguistique : des applications à la méthode ", in Flavie Fouchard, Adelaida Hermoso Mellado-Damas, Estefanía Marceteau Caballero, Marc Viémon, Adamantia Zerva et Alexia Zilliox (coords), La Recherche en études françaises : un éventail de possibilités. Séville, Editorial Universidad de Sevilla, 21-40.

Ducrot, Oswald (1972) : Dire et ne pas dire. Principes de sémantique. Paris, Hermann (coll. Savoir).

EDESO NATALÍAS, Verónica (2010) : "Valores de la interjección ay : desde el Tesoro de Covarrubias (1611) hasta el Diccionario de uso del español de América y España (2002) ", in María Teresa Encinas Manterola, Mónica González Manzano, Miguel Gutiérrez Maté, M.a Á. López Vallejo, Carolina Martín Gallego, Laura Romero Aguilera, Marta Torres Martínez et Irene Vicente Miguel (coords), Ars longa: diez años de AJIHLE, vol. 1. Buenos Aires, Voces del Sur, 237-250.

FrAISSE, Amel \& Patrick PAROUBEK (2015) : «Les interjections pour détecter les émotions », 
in Actes de la $22^{\circ}$ conférence sur le Traitement Automatique des Langues Naturelles (TALN 2015). URL : https://hal.archives-ouvertes.fr/hal-01617186/document.

FUENTES RODRÍGUEZ, Catalina (2018) : Diccionario de conectores y operadores del español. Madrid, Arco/Libros, $2^{\mathrm{e}}$ édition.

Fuentes RodríGUEZ, Catalina \& Esperanza R. ALCAIDE LARA (1996) : La expresión de la modalidad en el habla de Sevilla. Séville, Servicio de Publicaciones del Ayuntamiento de Sevilla.

FURUKAWA, Naoyo (2005) : "Heureusement qu'il est là : construction à élément thématique propositionnel ", in Naoyo Furukawa, Pour une sémantique des constructions grammaticales. Thème et thématicité. Bruxelles, De Boeck-Duculot, 123-134.

GONZÁLEZ RUIZ, Ramón \& Carmen LLAMAS SÁíZ [éds] (2011) : Gramática y discurso. Nuevas aportaciones sobre partículas discursivas del español. Pampelune, Eunsa.

GREENBAUM, Sidney (1969) : Studies in English Adverbial Usage. Londres, Longman.

GREVISSE, Maurice \& André GoOSSE (2008) : Le bon usage. Bruxelles, De Boeck-Duculot, $14^{\mathrm{e}}$ édition.

GUIMIER, C. (1998) : «Pourquoi peut-on dire Heureusement que Pierre est parti, mais pas *Malheureusement que Pierre est parti ?». Revue de Sémantique et Pragmatique, 3, 161 176.

HAILlET, Pierre Patrick (2013) : "Stratégie discursive : mise en relation de points de vue », in Jean-Claude Anscombre, María Luisa Donaire et Pierre Patrick Haillet (éds), Opérateurs discursifs du français. Eléments de description sémantique et pragmatique. Berne, Peter Lang, 33-36.

HuMmel, Martin (2012) : Polifuncionalidad, polisemia y estrategias retóricas. Los signos discursivos con base atributiva entre oralidad y escritura. Acerca del uso de bueno, claro, total, realmente, etc. Berlin / Boston, De Gruyter.

KLEIBER, Georges (2006) : "Sémiotique de l'interjection ». Langages, 1, 10-23.

LITTRÉ, Émile (sd) : Dictionnaire de la langue française. Paris, Hachette. URL : http://littre.reverso.net.

Maldonado, Concepción [dir] (1996) : Clave. Diccionario de uso del español actual. Madrid, SM.

MARTÍN ZORRAQUINO, María Antonia (1992) : "Partículas y modalidad », in Günter Holtus et al. (éds), Lexikon der Romanistichen Linguistik, VI, 1. Tubingue, Max Niemeyer Verlag, 110-124.

MARTín ZORRAQUINO, María Antonia (1999): «Aspectos de la gramática y de la pragmática de las partículas de modalidad en español actual », in Tomás Jiménez Juliá et al. (éds), Español como lengua extranjera: Enfoque comunicativo y gramática. Actas del IX Congreso de Asele. Saint-Jacques-de-Compostelle, Universidad de Santiago de Compostela, 2556. 
MARTÍN ZORRAQUiNO, María Antonia (2010): "Los marcadores del discurso y su morfología », in Esperanza Acín Villa et Óscar Loureda Lamas (eds), Los estudios sobre los marcadores del discurso, hoy. Madrid, Arco/Libros, 92-180.

MELÉNDEZ QUERO, Carlos (2009a) : Contribución al estudio de los adverbios disjuntos de valoración afectivo-emotiva en español actual. Saragosse, Université de Saragosse. URL: http://zaguan.unizar.es/record/2055.

MELÉNDEZ QUERO, Carlos (2009b) : "L'espagnol menos mal et ses traductions en français : analyse comparative d'un ensemble de particules modales ", Cahiers de lexicologie. Revue internationale de lexicologie et lexicographie, $95: 2$, 145-170.

MELÉNDEZ QUERO, Carlos (2015) : «La delimitación del significado de los adverbios evaluativos emotivos y su aprovechamiento en términos de estrategias discursivas ». Spanish in context, $12: 1$ [Bert Cornilie et Barbara de Cock, éds, Hearer-Orientation in Spoken Genres], 140-162.

MELÉNDEZ QUERO, Carlos (2016) : « La enseñanza de la gramática del discurso en la formación de hispanistas no nativos: el caso de los marcadores ". MarcoELE, 22. [Carmen Ballestero de Celis et Carlota Piedehierro Sáez, éds, Cuestiones de gramática para especialistas no nativos de español], 89-105. URL: http://marcoele.com/descargas/22/gramatica-espacialistas-no-nativos.pdf.

MELÉNDEZ QUERO, Carlos (2018) : «Remarques sur la traduction des adverbes de phrase évaluatifs émotifs ", in Carmen Ballestero de Celis et Yekaterina Garcia Markina (dirs.), L'épreuve de traduction au Capes et à l'Agrégation d'espagnol. Thème, version, choix de traduction. Paris, Armand Colin, 183-214.

MELÉNDEZ QUERO, Carlos (2019): «L'approche contrastive fondée sur des corpus écrits pour l'étude des particules discursives : le cas de hélas et ses équivalents en espagnol », in Flavie Fouchard, Adelaida Hermoso Mellado-Damas, Estefanía Marceteau Caballero, Marc Viémon, Adamantia Zerva et Alexia Zilliox (coords.), La Recherche en études françaises : un éventail de possibilités. Séville, Editorial Universidad de Sevilla, 589-597.

MOLINER, María (1966) : Diccionario de uso del español. Madrid, Gredos.

MOLINIER, Christian \& Françoise LEVRIER (2000) : Grammaire des adverbes : description des formes en-ment. Genève, Draz.

MøRDRUP, Ole (1976) : «Une analyse non-transformationnelle des adverbes en -ment». Revue Romane, 11, numéro spécial. Copenhague, Akademisk Forlag.

PORROCHE BALLESTEROS, Margarita (2009): Aspectos de la gramática del español coloquial para profesores de español como E/L2. Madrid, Arco/Libros.

PORROCHE BALLESTEROS, Margarita (2017) : «Sobre la partícula discursiva vaya ». Léxico Español Actual, 5, 187-207.

REAL ACADEMIA ESPaÑola (2009) : Nueva gramática de la lengua española. Madrid, Espasa.

REAL ACADEMia Española (sd) : Diccionario de la lengua española. Madrid, Espasa Calpe, $23^{\mathrm{e}}$ édition. URL : https://dle.rae.es/.

RIEGEL Martin ; Jean-Cristophe PELLAT \& René RIOUL (2009) : Grammaire méthodique $d u$ français. Paris, PUF, $9^{\mathrm{e}}$ édition. 
ROBERT, Paul (2010) : Le Nouveau Petit Robert. Paris, Dictionnaires le Robert.

RODRÍGUEZ SOMOLINOS, Amalia (2011) : "Présentation : Les marqueurs du discours - approches contrastives ". Langages, 184, 3-12.

ROSIER, Laurence (1995) : «L'interjection, partie honteuse du discours ». Scolia, 3, 109-121.

ROSIER, Laurence (1997) : «L'interjection comme point nodal de la créativité lexicale : la particule sensible Ä̈e ». Le moyen français, 39-40-41 [Autour de Jacques Monfrin. Néologie et création verbale : actes du colloque international, Université McGill, Montréal, 7-8-9 octobre], 451-460.

ROSIER, Laurence (2000) : "Interjection, subjectivité, expressivité et discours rapporté à l'écrit : petits effets d'un petit discours ». Cahiers de praxématique, 34, 19-50.

SANTOS Río, Luis (2003) : Diccionario de partículas. Salamanque, Luso Española de Ediciones.

SCHLYTER, Suzanne (1977) : La place des adverbs en -ment en français. [CD-ROM] Constance, Université de Constance.

SECO, Manuel; Olimpia ANDRÉS \& Gabino RAMOS (1999) : Diccionario del español actual. Madrid, Aguilar.

SIERRA SORIANO, Ascensión (2006) : «Interjections issues d'un verbe de mouvement : étude comparée français-espagnol ». Langages, 161, 73-90.

ŚWIĄTKOWSKA, Marcela (2006) : «L'interjection : entre deixis et anaphore ». Langages, 161, 47-56.

TANGHE, Sanne (2015) : «Prosodia y polifuncionalidad de los marcadores anda, vamos, vaya y venga ». Círculo de Lingüistica Aplicada a la Comunicación, 62, 125-147.

VANDERVEKEN, Daniel (1992) : "La théorie des actes du discours et l'analyse de la conversation ». Cahiers de linguistique française, 13, 9-62.

VÁZQUEZ MOLINA, Jesús Francisco (2019) : “Connecteurs, marqueurs, opérateurs... La notion d'opérateur discursif ", in Flavie Fouchard, Adelaida Hermoso Mellado-Damas, Estefanía Marceteau Caballero, Marc Viémon, Adamantia Zerva et Alexia Zilliox (coords.), La Recherche en études françaises : un éventail de possibilités. Séville, Editorial Universidad de Sevilla, 683-691.

VINCENSINI, Jean-Jacques (2006) : «Formes et fonctions structurantes. À propos de quelques interjections en ancien et en moyen français ». Langages, 161, 101-111. 